\title{
Plasmon-Enhanced Fluorescence Biosensors: a Review
}

\author{
Martin Bauch • Koji Toma • Mana Toma • \\ Qingwen Zhang • Jakub Dostalek
}

Received: 22 November 2013 / Accepted: 6 December 2013 /Published online: 28 December 2013

(C) Springer Science+Business Media New York 2013

\begin{abstract}
Surfaces of metallic films and metallic nanoparticles can strongly confine electromagnetic field through its coupling to propagating or localized surface plasmons. This interaction is associated with large enhancement of the field intensity and local optical density of states which provides means to increase excitation rate, raise quantum yield, and control far field angular distribution of fluorescence light emitted by organic dyes and quantum dots. Such emitters are commonly used as labels in assays for detection of chemical and biological species. Their interaction with surface plasmons allows amplifying fluorescence signal (brightness) that accompanies molecular binding events by several orders of magnitude. In conjunction with interfacial architectures for the specific capture of target analyte on a metallic surface, plasmon-enhanced fluorescence (PEF) that is also referred to as metal-enhanced fluorescence (MEF) represents an attractive method for shortening detection times and increasing sensitivity of various fluorescence-based analytical technologies. This review provides an introduction to fundamentals of PEF, illustrates current developments in design of metallic nanostructures for efficient fluorescence signal amplification that utilizes propagating and localized surface plasmons, and summarizes current implementations to biosensors for
\end{abstract}

\footnotetext{
M. Bauch $\cdot$ K. Toma $\cdot$ M. Toma $\cdot$ Q. Zhang $\cdot$ J. Dostalek $(\bowtie)$

AIT-Austrian Institute of Technology GmbH, Muthgasse 11, Vienna 1190, Austria

e-mail: jakub.dostalek@ait.ac.at

URL: www.ait.ac.at

Present Address:

K. Toma $\cdot$ M. Toma

Forschungszentrum Jülich GmbH, Jülich 52425, Germany

Present Address:

Q. Zhang

Department of Physical Chemistry, School of Chemistry, BIT-Beijing Institute of Technology, Beijing 100081, China
}

detection of trace amounts of biomarkers, toxins, and pathogens that are relevant to medical diagnostics and food control.

Keywords Surface plasmon resonance $\cdot$ Plasmonics . Fluorescence · Plasmon-enhanced fluorescence . Metal-enhanced fluorescence $\cdot$ Biosensor

\section{Introduction}

Research in plasmonic confinement of light to volumes much smaller than wavelength paved new routes to powerful amplification schemes in optical spectroscopies. In particular, we witnessed rapid advancements in surface-enhanced Raman spectroscopy (SERS), surface-enhanced infrared spectroscopy (SEIRA), and surface plasmon-enhanced fluorescence spectroscopy (PEF) [1-5] over the last years. This progress was accompanied with the implementation of plasmonics to a range of analytical technologies for the detection of chemical and biological species that are relevant to important areas of medical diagnostics, food control, and security [6, 7]. Among these, fluorescence is arguably the mostly spread optical method, and it has been already routinely used for readout of assays over several decades. In PEF, fluorophore labels are coupled with the tightly confined field of surface plasmons collective oscillation of charge density and associated electromagnetic field on a surface of metallic films and nanostructures. This interaction can be engineered to dramatically enhance emitted fluorescence light intensity which is desired for detecting minute amounts of analytes with improved limit of detection and shorten analysis time. PEF was subject to a number of excellent reviews over the last years covering the fundamental research on the interaction of nanoscale emitters with metallic surfaces [8-10] as well as its implementation into advanced assays and applications for biological studies $[4,5,11-13]$. This paper aims at updating these reviews and 
providing key leads for a design of plasmonic nanostructures for efficient amplification on realistic biochips. Firstly, fundamentals of surface plasmon-fluorophore interactions are introduced, and the performance characteristics of metallic nanostructures that are essential for strong enhancement of fluorescence signal are discussed. Afterwards, implementations of PEF biosensor devices for rapid detection of trace amounts of biomarkers and harmful compounds including toxins and pathogens are reviewed.

\section{Interaction of Fluorophores with Surface Plasmons}

The coupling of light with localized surface plasmons (LSPs - supported by metallic nanoparticles) and surface plasmon polaritons (SPPs - traveling along continuous metallic films) can provide strong confinement of electromagnetic field intensity. These fields can interact with fluorophores at their absorption $\lambda_{\mathrm{ab}}$ and emission $\lambda_{\mathrm{em}}$ wavelengths which alter respective transitions between the ground state and higher excited states (see Fig. 1).

Surface plasmon-induced changes in the excitation and decay rates can be classically described by Maxwell equations by using fluorophore absorption $\mu_{\mathrm{ab}}$ and emission $\mu_{\mathrm{em}}$ electric dipole moments [8]. The excitation rate of a fluorophore $\gamma_{\mathrm{e}}$ that is irradiated by an incident wave with the electric field $E$ at the absorption wavelength $\lambda_{\mathrm{ab}}$ can be expressed as

$\gamma_{\mathrm{e}^{\propto}}\left|E \cdot \mu_{\mathrm{ab}}\right|^{2}$

Let us note that Eq. (1) holds for small amplitudes of electric field $E$ for which the excitation rate is far from saturation. After its excitation, the fluorophore can return to its ground state by emitting a photon at a higher wavelength $\lambda_{\mathrm{em}}$ (radiative decay rate $\gamma_{\mathrm{r}}$ ) or without emitting a photon (nonradiative decay rate $\gamma_{\text {nr }}$ ). Further, we denote an intrinsic radiative decay rate as $\gamma_{\mathrm{r}}^{0}$, nonradiative decay rate as $\gamma_{\mathrm{nr}}^{0}$, and quantum yield $\eta_{0}=\gamma_{\mathrm{r}}^{0} /\left(\gamma_{\mathrm{r}}^{0}+\gamma_{\mathrm{nr}}^{0}\right)$ for a fluorophore in a homogenous aqueous environment. When the fluorophore is brought in the vicinity of a metallic structure, the radiative decay rate $\gamma_{\mathrm{r}}$ and nonradiative decay rate $\gamma_{\mathrm{nr}}=\gamma_{\mathrm{nr}}^{0}+\gamma_{\mathrm{abs}}$ are changed due to the increased local density of optical states (LDOS) at $\lambda_{\mathrm{em}}$ that is associated with plasmon-enhanced field intensity $|E|^{2}$. This leads to the modified quantum yield $\eta[14]$ :

$\eta=\frac{\gamma_{\mathrm{r}} / \gamma_{\mathrm{r}}^{0}}{\gamma_{\mathrm{r}} / \gamma_{\mathrm{r}}^{0}+\gamma_{\mathrm{abs}} / \gamma_{\mathrm{r}}^{0}+\left(1-\eta^{0}\right) / \eta^{0}}$

For short distances from the metal surface $d<15 \mathrm{~nm}$, strong quenching of radiative transitions occurs due to Förster energy transfer between a fluorophore and a metal. This quenching is accompanied with metal-enhanced nonradiative decay rate a

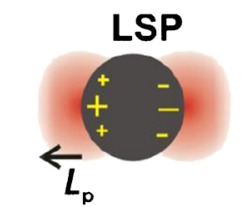

Fluorophore
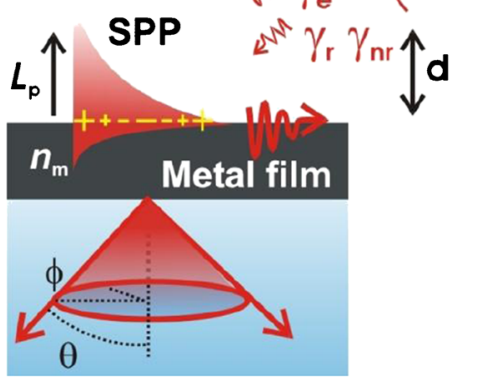

b

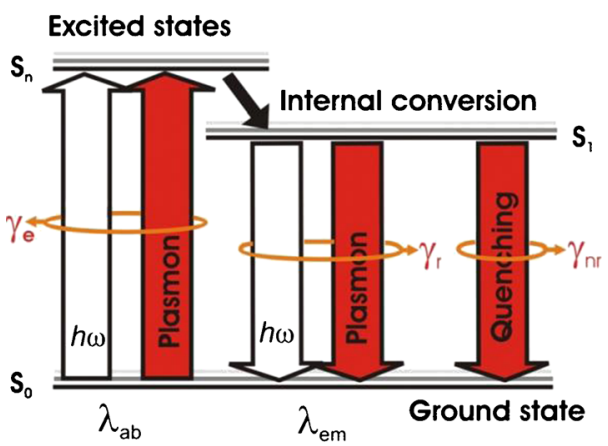

Fig. 1 a Schematic of confined field of SPP and LSP modes coupled with a fluorophore and b Jablonski diagram showing surface plasmonmediated transitions between the fluorophore ground state and higher excited states

$\gamma_{\mathrm{abs}}$ that competes with $\gamma_{\mathrm{r}}$, shortens the lifetime of the fluorophore excited state $\tau=1 /\left(\gamma_{\mathrm{r}}+\gamma_{\mathrm{nr}}\right)$, and decreases the quantum yield $\eta$. At longer distances $d$ that are below the decay length of surface plasmon field $L_{\mathrm{p}}$, the emission via surface plasmons becomes dominant. When these surface plasmons are out-coupled to the far field, such interaction can enhance the radiative decay rate $\gamma_{\mathrm{r}}$ and thus increase quantum yield $\eta$. As Fig. 2 illustrates, this effect is particularly strong for fluorophores with low intrinsic quantum yield $\eta_{0}$. For instance, the factor of $\eta / \eta_{0} \sim 4$ was calculated at the distance of $d=10 \mathrm{~nm}$ from a gold disk nanoparticle and a fluorophore with $\eta_{0}=0.05$. For a flat metallic surface, lower enhancement of the quantum yield is observed owing to the weaker field confinement of SPPs compared to LSPs. At a distance $d \gg L_{\mathrm{p}}$, the emission from fluorophores is decoupled from surface plasmons and becomes only weakly affected by the interference with waves back-reflected from the metal surface [15].

Let us note that the emission via dipolar LSP modes on metallic nanoparticles is directly converted to the far field via scattering, and thus, it contributes to $\gamma_{\mathrm{r}}$. However, the emission via SPPs traveling along continuous metallic surfaces requires 


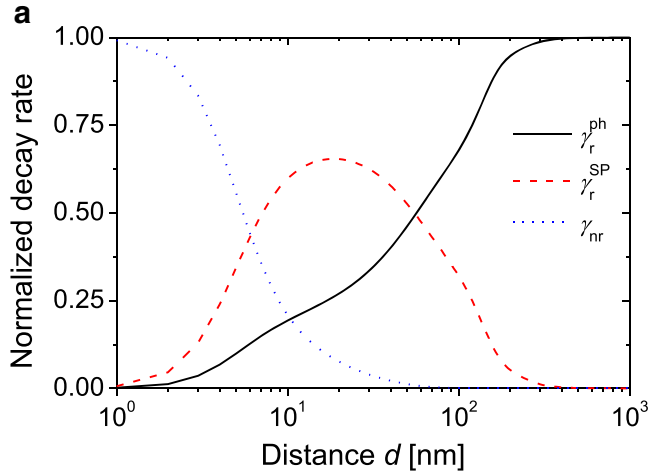

b

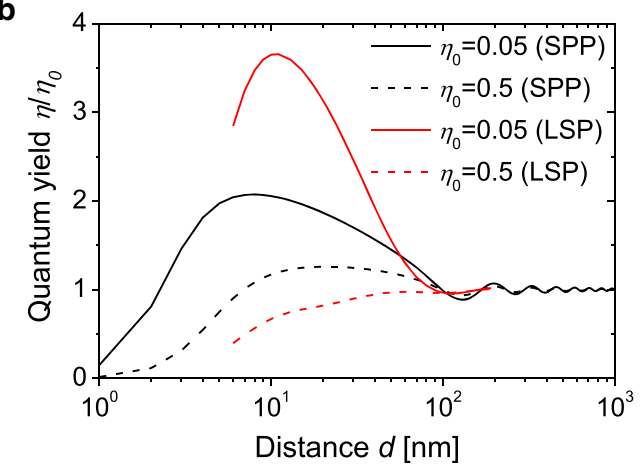

Fig. 2 a Simulated radiative rate $\gamma_{\mathbf{r}}$ (associated with emission to far field $\gamma_{\mathbf{r}}^{\mathbf{p h}}$ and via surface plasmons $\boldsymbol{\gamma}_{\mathbf{r}}^{\mathbf{S P}}$ ) and nonradiative rate $\gamma_{\mathbf{n r}}$ and $\mathbf{b}$ respective changes in a quantum yield $\eta$ for a fluorophore with low $\eta_{0}=$ 0.05 and high $\eta_{0}=0.5$ intrinsic quantum yield. The rates were normalized by the total decay rate $\gamma_{\mathbf{r}}^{\mathbf{p h}}+\gamma_{\mathbf{r}}^{\mathbf{S P}}+\gamma_{\mathbf{n r}}$ A flat gold surface supporting SPPs and gold disk nanoparticle with a diameter of $D=110 \mathrm{~nm}$ and height of $50 \mathrm{~nm}$ supporting LSP were assumed. Simulations were carried out for a randomly oriented fluorophore in water and the emission wavelength of $\lambda_{\mathrm{em}}=670 \mathrm{~nm}$

an additional coupler in order to extract such emitted radiation. Similar to surface plasmon resonance (SPR) spectrometers, reverse Kretschmann configuration of attenuated total internal reflection (ATR) method or diffraction on periodically corrugated metallic surface (grating) can be used. As Fig. 1a shows for the reverse Kretschmann configuration, the emission via SPPs is cross-coupled through a thin metallic film and forms a characteristic cone propagating in a high refractive index dielectric substrate $[8,16]$. The fluorescence light cone is centered at the polar SPR angle $\theta$ for which SPPs on the top metal surface are phase-matched with optical waves in the dielectric substrate (see Fig. 3a). Similarly, diffractioncoupling of SPPs to propagating waves is possible through additional momentum provided by a periodic grating which allows for concentrating the emitted light towards a specific direction (see Fig. 3b).

The ability to control the emission angular distribution offers attractive means to increase the collecting efficiency of fluorescence light in fluorescence devices by its "beaming" towards a detector. Moreover, the highly directional fluorescence emission is useful for suppressing background signal
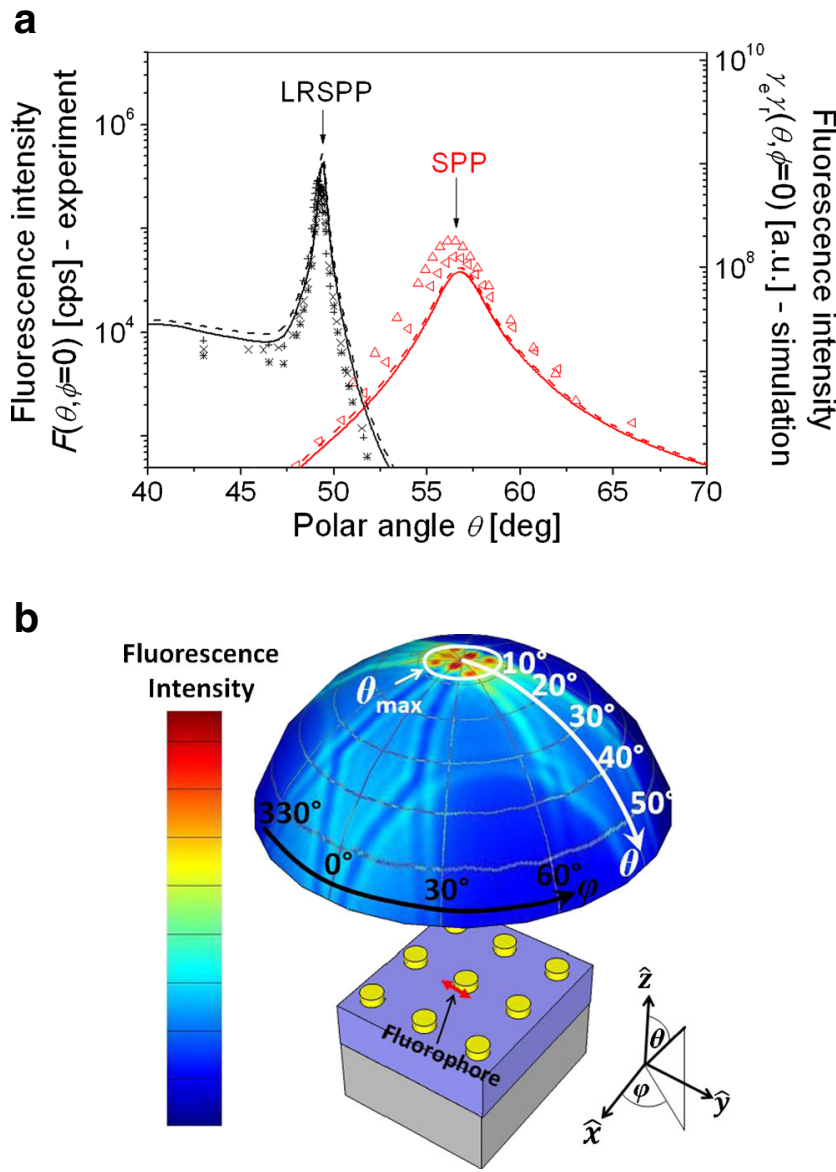

Fig. 3 a Simulated and experimental angular dependence of surface plasmon-coupled emission via regular surface plasmon polaritons (SPPs) and long-range surface plasmon polaritons (LRSPPs) with reverse Kretschmann configuration. b Angular distribution of emitted light from a dipole coupled with arrays of metallic nanoparticles supporting collective localized surface plasmons (reproduced with permission from [65] and [53])

that originates from (typically isotropic) scattering and autofluorescence. For the majority of fluorescence detection schemes, less than a few percent of emitted photons is delivered to a detector. As illustrated in Fig. 3, most of the emitted radiation intensity can be emitted via surface plasmons and subsequently out-coupled to a specific angle. The directionality of surface plasmon-coupled emission can be quantified by the following factor $f[17-19]$ :

$f=\max \left\{\frac{4 \pi \gamma_{\mathrm{r}}(\theta, \phi)}{\iint \gamma_{\mathrm{r}}(\theta, \phi) \sin (\theta) d \theta d \phi}\right\}$

where $\gamma_{\mathrm{r}}(\theta, \phi)$ is the radiative decay rate density at $\lambda_{\mathrm{em}}$ that is integrated over all polar $\theta$ and azimuthal $\phi$ angles in the denominator.

In summary, the coupling of fluorophores with surface plasmons on metallic surfaces allows amplifying the intensity 
of detected fluorescence light by the combination of three effects-(1) increasing the excitation rate $\gamma_{e}$ through the plasmon-enhanced field intensity at the absorption wavelength of $\lambda_{\mathrm{ab}}$, (2) enhancing fluorophore quantum yield $\eta$, and (3) high directionality $f$ of plasmon-coupled emission at the wavelength $\lambda_{\mathrm{em}}$ :

$\mathrm{EF} \propto \frac{\gamma_{\mathrm{e}}}{\gamma_{\mathrm{e}}^{0}} \times \frac{\eta}{\eta^{0}} \times f$

where $\mathrm{EF}$ is the enhancement factor of detected fluorescence intensity with respect to that measured without the metallic structures (e.g., a free fluorophore in homogenous aqueous environment). Let us note that the enhancement factor strongly depends on the fluorophore orientation due to the polarization sensitivity of surface plasmon resonance. As the orientation of fluorophores is typically random, the enhancement factor measured for an ensemble of emitters is averaged across all possible orientations of the absorption and emission dipole moments $\mu_{\mathrm{ab}}$ and $\mu_{\mathrm{em}}$, respectively. In addition, the PEF amplification is highly surface sensitive and occurs only at distances $d$ below the surface plasmon probing depth $L_{\mathrm{p}}$. Therefore, it can provide means to better distinguish between specific fluorescence signal and background that originate from bulk effects including auto-fluorescence or scattering.

\section{Surface Plasmon Field Intensity Enhancement}

PEF is directly related to the strength of the field $E$ generated in the vicinity of metallic surfaces. Therefore, the design of metallic nanostructures providing maximum field intensity enhancement upon the excitation of surface plasmons is of key importance. Various materials exhibit plasmonic characteristics including noble metals, transparent conducting oxides, graphene, and semiconductors [20]. Among these, noble metals are preferably used for PEF as they support surface plasmons in the visible and near infrared part of the spectrum, and they exhibit low damping associated with inter- and intraband transitions. The electromagnetic field intensity enhancement $|E|^{2} /\left|E_{0}\right|^{2}$ that is accompanied with the coupling to surface plasmons strongly depends on the (complex) metal refractive index $n_{\mathrm{m}}$. For LSPs, one can show that the field enhancement is approximately proportional to the figure of merit $\left|\operatorname{Re}\left\{n_{\mathrm{m}}^{2}\right\}\right| /$ $\operatorname{Im}\left\{n_{\mathrm{m}}^{2}\right\}$. The coupling to SPPs on a continuous film is accompanied with the field enhancement that scales with a similar term $\left(\operatorname{Re}\left\{n_{\mathrm{m}}^{2}\right\}\right)^{2} / \operatorname{Im}\left\{n_{\mathrm{m}}^{2}\right\}$. The SPP figure of merit is plotted for gold, silver, and aluminum in Fig. 4 and shows that aluminum can be the preferable metal of choice for PEF at wavelengths in the blue and UV region [21]. In the visible and near infrared part of the spectrum, surface plasmons on silver and gold surfaces provide higher field enhancement which increases

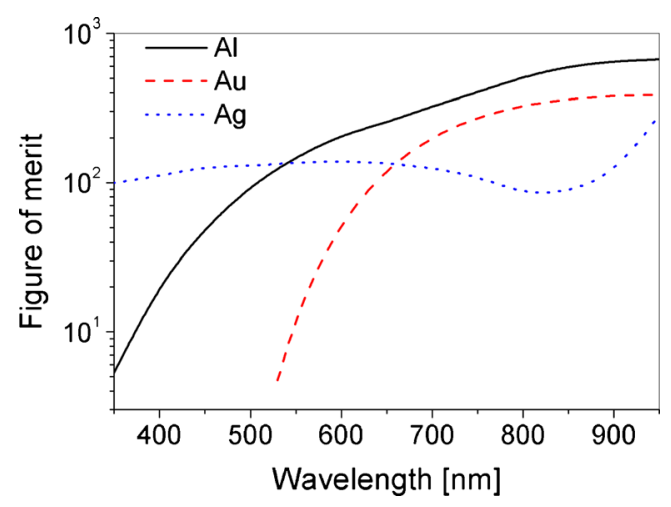

Fig. 4 Simulated figure of merit for the plasmon-enhanced field intensity associated with the excitation of SPPs on Al, Au, and Ag surfaces

with the wavelength. Silver is known to provide stronger field intensity enhancement than gold (particularly at wavelengths $\lambda<600 \mathrm{~nm}$ ); however, gold is more often used due to its better chemical stability.

Further, the field intensity enhancement $|E|^{2} /\left|E_{0}\right|^{2}$ that is associated with the excitation of SPPs and LSPs on most commonly used metallic nanostructures is discussed. As the field intensity enhancement factors are difficult to measure directly, we provide an overview of $|E|^{2} /\left|E_{0}\right|^{2}$ values obtained from simulations (a brief summary can be found in Table 1). We preferably selected works where the near-field simulations are supported by experimentally obtained data on far-field properties of studied metallic nanostructures. Let us note that further detailed information on plasmonic properties of metallic nanostructures can be found in numerous specialized review papers [22-26].

\section{Continuous Metallic Films}

Characteristics of SPP modes traveling along metallic surfaces can be tuned by their mutual interaction. For instance, a thin metallic film supports SPP modes at each of its two surfaces. These modes become coupled when the thickness of the metal film $d_{\mathrm{m}}$ is comparable with the plasmon penetration depth into the metal (typically up to $10 \mathrm{~nm}$ ) and when the film is surrounded by dielectrics with similar refractive indices (as shown in the respective figure in Table 1). The spatial overlap and phase matching between SPPs leads to the establishing of coupled symmetrical and antisymmetrical surface plasmon polariton modes [27]. The mode with the antisymmetrical profile of the parallel component of the electric field $E_{\|}$is referred to as long range surface plasmon polariton (LRSPP) while the one with the symmetrical profile is short-range surface plasmon polariton (SRSPP). LRSPPs are weaker guided by the metal film than regular SPPs, and thus, they can propagate to longer distances and exhibit decreased Ohmic losses, and their field probes to larger distances $L_{\mathrm{p}}$ from the metal surface. Another type of coupled SPP mode can be excited on metallic surfaces with dense subdiffractive gratings 
Table 1 Comparison of the field intensity enhancement at a distance from the metallic surface of $d \sim 10-20 \mathrm{~nm}$ for selected nanostructures supporting SPP and LSP modes. Figures reprinted with permission from references indicated on the right side

\begin{tabular}{|c|c|c|c|c|c|}
\hline Plasmonic Structure & Schematics & Fabrication & Wavelength & $|\mathrm{E}|^{2} /|\mathrm{E} 0|^{2}$ & Ref. \\
\hline $\begin{array}{l}\text { LRSPP: Flat and } \\
\text { corrugated Au films }\end{array}$ & & $\begin{array}{l}\text { Nanoimprint } \\
\text { lithography }\end{array}$ & $\lambda=630 \mathrm{~nm}$ & $\sim 90$ & \\
\hline $\begin{array}{l}\text { SPP: Flat and corrugated } \\
\text { Au films }\end{array}$ & & & $\lambda=630 \mathrm{~nm}$ & $\sim 45$ & \\
\hline $\begin{array}{l}\text { LSP: Au nanoshell and } \\
\text { spherical nanoparticles }\end{array}$ & & $\begin{array}{l}\text { Chemical } \\
\text { synthesis }\end{array}$ & $\lambda=617 \mathrm{~nm}$ & $\sim 10$ & [37] \\
\hline $\begin{array}{l}\text { LSP: Au nanoparticle } \\
\text { dimers }\end{array}$ & & $\begin{array}{l}\text { Electron beam } \\
\text { and focused ion } \\
\text { beam lithography }\end{array}$ & $\begin{array}{l}\lambda=780 \mathrm{~nm} \\
\lambda=780 \mathrm{~nm} \\
\lambda=630 \mathrm{~nm}\end{array}$ & $\begin{array}{l}181 \\
\sim 350 \\
\sim 100\end{array}$ & $\begin{array}{l}{[43]} \\
{[44]} \\
{[57]}\end{array}$ \\
\hline $\begin{array}{l}\text { SPP and LSP: Au } \\
\text { nanodisk and nanoholes } \\
\text { arrays }\end{array}$ & & $\begin{array}{l}\text { Electron beam } \\
\text { and focused ion } \\
\text { beam lithography }\end{array}$ & $\lambda \sim 630 \mathrm{~nm}$ & $\sim 10$ & [46] \\
\hline $\begin{array}{l}\text { LSP: Ag bow tie NP } \\
\text { arrays, LSP }\end{array}$ & & $\begin{array}{l}\text { Colloidal } \\
\text { lithography }\end{array}$ & $\lambda=780 \mathrm{~nm}$ & $\sim 100$ & [48] \\
\hline $\begin{array}{l}\text { SPP and LSP: Concentric } \\
\text { gratings with nanohole }\end{array}$ & & $\begin{array}{l}\text { Electron beam } \\
\text { and focused ion } \\
\text { beam lithography }\end{array}$ & $\lambda=633 \mathrm{~nm}$ & $\sim 10$ & {$[34,69]$} \\
\hline $\begin{array}{l}\text { cLSP and SPP: } \\
\text { Diffractive arrays of } \\
\text { cylindrical nanoparticles }\end{array}$ & & $\begin{array}{l}\text { Interference } \\
\text { lithography }\end{array}$ & $\lambda=630-670 \mathrm{~nm}$ & $\sim 200$ & [53] \\
\hline
\end{tabular}

[28]. Diffraction on such periodic modulation let counterpropagating SPPs interact which opens a bandgap in the SPP dispersion relation. Two Bragg-scattered surface plasmon polariton (BSSPP) modes occur at edges of the bandgap with the field intensity localized either in grating valleys or at peeks of the periodic modulation.

The coupling to SPP-like modes provides field intensity enhancement $|E|^{2} /\left|E_{0}\right|^{2}$ that exponentially decays from the metal surface. As calculated in Fig. 5 for a gold surface and distance of $d=15 \mathrm{~nm}$, the field intensity enhancement $|E|^{2} /\left|E_{0}\right|^{2}$ increases with the wavelength and follows the dependence of the figure of merit presented in Fig. 4. The enhancement for ATR and diffraction grating-based SPP couplers is similar, and it reaches $|E|^{2} /\left|E_{0}\right|^{2} \sim 10$ at $\lambda=550 \mathrm{~nm}$ and $\sim 85$ at $\lambda=$ $900 \mathrm{~nm}$. The excitation of LRSPP modes on a gold film with $d_{\mathrm{m}}=20 \mathrm{~nm}$ is accompanied with an enhancement that is stronger by a factor of 3-5 and allows reaching significantly longer distances $L_{\mathrm{p}}$ with respect to regular SPPs. The behavior of BSSPP modes is analogical to LRSPPs and SRSPPs and exhibits similar features [29].

In order to further boost the field intensity enhancement up, the field of SPPs can be confined in the direction parallel to the surface. A continuous metal film that is perforated by arrays of nanoholes (see the respective figure in Table 1) represents a well-characterized system $[30,31]$ that can act as a diffraction grating for the excitation of SPPs and at the same time supports laterally confined LSPs. In a different example, finite difference time domain (FDTD) simulations were carried out for the metallic grating with narrow, high-aspect-ratio grooves enabling diffraction-based excitation of SPPs that interact with LSP modes at the grooves [32]. This work predicted large field intensity enhancement of $|E|^{2} /\left|E_{0}\right|^{2} \sim 10^{3}$ at LSP

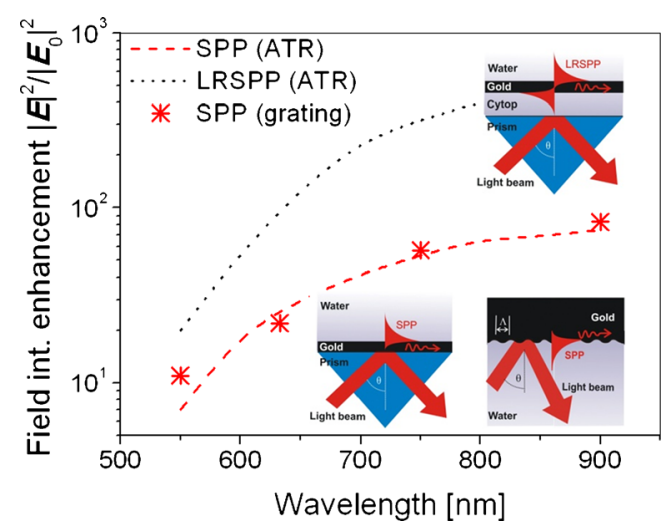

Fig. 5 Simulated field intensity enhancement at the distance of $d=15 \mathrm{~nm}$ for the full coupling efficiency to SPPs (diffraction grating - stars and Kretschmann geometry - dashed line) and LRSPP (Kretschmann geometry - dotted line). The thicknesses of a gold film of $d_{\mathrm{m}}=50$ and $20 \mathrm{~nm}$ were assumed for Kretschmann configuration for SPP and LRSPP modes, respectively. For the diffraction-based coupling, the period and modulation depth of sinusoidal grating was adjusted for normal incidence excitation 
wavelength of $\lambda=820 \mathrm{~nm}$ for a gold grating structure with 60 $\mathrm{nm}$-wide and 90-nm-deep grooves arranged with a period of $\Lambda=560 \mathrm{~nm}$. Another approach that takes advantage of the interplay between SPP and LSP modes utilized a relief concentric grating with a narrow hole in its center (see the respective figure in Table 1) [33, 34]. FDTD analysis of a silver film with five concentric grooves (period $\Lambda=440 \mathrm{~nm}$ ) surrounding a nanohole (diameter of $140 \mathrm{~nm}$ ) showed a field enhancement of $|E|^{2} /\left|E_{0}\right|^{2} \sim 40$ that was associated with the focusing of SPPs to the central nanohole supporting LSPs at the wavelength $\lambda=585 \mathrm{~nm}$ [33].

\section{Metallic Nanoparticles}

The plasmonic structure that has arguably become the most investigated in detail is the spherical metallic nanoparticle. If its diameter $D$ is much smaller than the resonant wavelength $\lambda$, it supports only a dipole LSP mode with the field intensity decreasing away from the metal as $\sim(D /[0.5 D+d])^{3}[35]$. This formula gives an estimate of the probing depth LSP field that roughly scales with the particle diameter $L_{\mathrm{p}} \sim D$. The excitation of LSPs on a gold spherical nanoparticle immersed in water provides a moderate maximum field intensity enhancement of $|E|^{2} /\left|E_{0}\right|^{2} \sim 18$ as calculated for $D=20 \mathrm{~nm}$ at $\lambda=521 \mathrm{~nm}$. Localized surface plasmon resonance (LSPR) occurs at higher wavelengths on nanoparticles with a thin metallic shell capping a spherical dielectric core (nanoshell particles - see the respective figure in Table 1). The interaction of LSP modes at the inner and outer metal surfaces red shifts the LSPR wavelength and allows reaching higher field intensity strength [36]. For instance, a nanoshell nanoparticle with the outer diameter of $D=54 \mathrm{~nm}$ and gold layer thickness of $14 \mathrm{~nm}$ was shown to enhance the field intensity by a factor of $\left.\left|E^{2} /\right| E_{0}\right|^{2} \sim 10^{2}$ at the resonant wavelength $\lambda=617 \mathrm{~nm}$ [37]. Nanoparticles with decreased symmetry support multiple LSP modes at different wavelengths. For example, elongated rod metallic nanoparticles support LSP modes with a dipole moment oscillating parallel and perpendicular to the nanoparticle axis [38]. Higher enhancement occurs for the excitation of LSP with the parallel dipole moment which concentrates the field intensity at nanoparticle tips. For instance, a gold rod nanoparticle with a length of $77 \mathrm{~nm}$ and a diameter of $28 \mathrm{~nm}$ was reported to enhance the field intensity by a factor of $|E|^{2} /\left|E_{0}\right|^{2} \sim 10^{2}[39]$ at the resonant wavelength $\lambda=780 \mathrm{~nm}$. In general, sharper metallic tips allow for more efficient concentrating of the light intensity. For example, gold triangle nanoparticles with a side length of $100 \mathrm{~nm}$ and a height of $20 \mathrm{~nm}$ were predicted to provide the field intensity enhancement $|E|^{2} /\left|E_{0}\right|^{2}>10^{3}$ at the resonant wavelength $\lambda=514 \mathrm{~nm}$ [40]. However, let us note that such field enhancement strongly decreases with increasing tip curvature and distance $d$ from the metal. Therefore, the field intensity enhancement that can be experimentally achieved at distances $d$ relevant to PEF is typically significantly lower.

\section{Metallic Nanoparticle Dimers}

Individual nanoparticles can serve as building blocks for the design of more complex metallic nanostructures with controlled LSPR properties. Near-field interaction of two spherical metallic nanoparticles brought in close proximity (nanoparticle dimer) leads to an establishment of a new LSP mode with a dipole moment aligned parallel to the dimer axis. This mode strongly confines the field intensity in the gap. For example, the maximum field enhancement of $|E|^{2} /\left|E_{0}\right|^{2}$ $\sim 1.8 \times 10^{3}$ was simulated by FDTD method for a gap LSP mode at a wavelength of $\lambda=633 \mathrm{~nm}$ that was supported by gold nanoparticles with a diameter of $D=30 \mathrm{~nm}$ and gap width of $3 \mathrm{~nm}$ [41]. Two end-to-end oriented gold rod nanoparticles were predicted to enhance the field intensity by a higher factor of $|E|^{2} /\left|E_{0}\right|^{2} \sim 10^{4}$ for a dimer gap width of $1 \mathrm{~nm}$ and resonant wavelength between $\lambda=700$ and $800 \mathrm{~nm}[38,42]$. The employment of triangular nanoparticle dimers with sharp tips oriented towards each other allows for even tighter confinement of the field intensity. This system is referred as to "bow tie" nanoantenna (see the respective figure in Table 1). Green's tensor-based model predicted the field enhancement $|E|^{2} /\left|E_{0}\right|^{2}$ $>10^{3}$ for a bow tie nanoparticle with a gap width of a few nanometers and LSPR wavelength at $\lambda \sim 800 \mathrm{~nm}$ [42]. The enhancement rapidly drops with increasing gap width. For instance, the enhancement factor of $|E|^{2} /\left|E_{0}\right|^{2}=2-3 \times 10^{2}$ was simulated for the gold bow tie nanoparticle with a gap width of $\sim 20 \mathrm{~nm}$ and realistic tip curvature at a similar resonant wavelength [43]. It should be noted that the majority of studies describe idealized nanoparticle geometries, and we witnessed only recently simulations that take into account their roughness and shape irregularities [44].

\section{Metallic Nanoparticle Arrays}

Periodic arrays of metallic nanoparticles enable enhancing the field intensity through long- and short-distance coupling of LSPs supported by individual nanoparticles [25]. For distances between nanoparticles that are close to the wavelength of incident light, long-distance (diffraction) interaction dominates and it is typically manifested as narrowing of the LSPR absorption band [45]. For short distances that are comparable with the decay length of LSPs $L_{\mathrm{p}}$, near-field interaction of LSPs builds up which is accompanied by a shift of LSPR wavelengths and altered field intensity profile in the vicinity of the nanoparticles. For near-field interaction with the gap width between plasmonic nanoparticles $>10 \mathrm{~nm}$, typically only moderate enhancement occurs. For instance, $\left.\left|E^{2} /\right| E_{0}\right|^{2}$ $\sim 10$ was reported for dense rectangular square arrays of gold disk nanoparticles $[46,47]$ at wavelengths of $530-630 \mathrm{~nm}$. 
Similarly, an inverse structure of densely packed nondiffractive arrays of nanoholes yields intensity enhancements of $|E|^{2} /\left|E_{0}\right|^{2} \sim 16$ at wavelength of $\lambda=600 \mathrm{~nm}$ [46]. The LSP field strength can be increased by using arrays of sharp nanoparticles such as nanotriangles that are arranged in a structure that resembles a bow tie nano-antenna. For instance, field enhancement of $|E|^{2} /\left|E_{0}\right|^{2} \sim 10^{2}$ at $\lambda=780 \mathrm{~nm}$ has been simulated for closely packed arrays of silver triangle nanoparticles by FDTD [48].

Diffractive coupling between metallic nanoparticles provides an alternative mechanism to achieve larger LSP field intensity enhancements. Such interaction gives rise to collective (lattice) localized surface plasmons (cLSPs), and it should be noted that this type of interaction is particularly strong for symmetrical geometry (i.e., the refractive index above and below the arrays is the same) [49]. It origins from phase matching of LSPs at wavelengths that coincide with the LSPR band of individual nanoparticles. With respect to regular LSPs, collective localized surface plasmons trap light at a surface more efficiently and exhibit decreased radiative damping which consequently leads to strong enhancements [45, 50-52]. FDTD simulations of cLSP arrays of gold disk nanoparticles showed more than tenfold increased field strength compared to identical individual LSP nanoparticles [53]. The same work predicted the enhancement of $|E|^{2} /\left|E_{0}\right|^{2}=$ $2 \times 10^{2}$ for cLSPs at wavelengths $\lambda=630-670 \mathrm{~nm}$ and relatively large distance of $d=20 \mathrm{~nm}$.

\section{Plasmon-Enhanced Fluorescence}

Even though early investigations on surface plasmonmediated fluorescence date several decades back [54, 55], we currently witnessed a rapidly increasing number studies on this phenomenon that were performed on ensembles of fluorophores and more recently also for individual fluorophores $[14,56]$. These efforts resulted in the development of plasmonic structures that enhance the fluorescence intensity over three orders of magnitude $\mathrm{EF}>10^{3}[43,57]$. This section is devoted to the deconvoluting of key factors acting in efficient PEF. We particularly focus on the choice of a metallic nanostructure that determines the strength of surface plasmon field $E$, spectral overlap of surface plasmon resonances with fluorophore excitation and/or emission wavelengths, orientation and intrinsic quantum yield of fluorophores, and methods for the extracting of surface plasmon-coupled emission from a surface to the far field. A comparison of PEF performance characteristics for selected plasmonic structures is presented in Table 2. Let us note that if not stated differently, the discussed studies were performed with ensembles of dye molecules randomly attached to the top of a spacer layer that controls the distance from the metal $d$.

\section{Flat Continuous Metallic Films}

SPPs on continuous metallic surfaces were mostly used for enhancing the excitation field strength at $\lambda_{\mathrm{ab}}$ and for exploiting the surface plasmon-driven emission at $\lambda_{\mathrm{em}}$. The fluorescence signal increase of $\mathrm{EF}=32$ was measured for the excitation of high quantum yield rhodamine- $6 \mathrm{G}$ dye $\left(\eta_{0}=0.95, \lambda_{\mathrm{ab}}=530 \mathrm{~nm}\right.$, $\lambda_{\mathrm{em}}=550 \mathrm{~nm}$ ) via SPPs at a distance of about $d \sim 10 \mathrm{~nm}$ [58]. In this work, Kretschmann configuration with a thin silver film was used to generate SPPs at a wavelength of $543 \mathrm{~nm}$. A similar value was obtained for medium quantum yield Cy5 dye $\left(\eta_{0}=\right.$ $0.28, \lambda_{\mathrm{ab}}=640 \mathrm{~nm}, \lambda_{\mathrm{em}}=670 \mathrm{~nm}$ ) that was probed by SPPs on a gold surface at a higher wavelength of $\lambda=633 \mathrm{~nm}$ [11]. Layer structures that support LRSPPs allow the further increase of the excitation strength at $\lambda_{\mathrm{ab}}$ owing to smaller damping of these modes and associated stronger field intensity [59]. For Cy5 dyes attached onto a gold surface at a distance $d=15-20 \mathrm{~nm}$, an additional two- to threefold increment of fluorescence intensity was reported compared to that for regular SPPs [60, 61]. These values are lower than the field intensity enhancement predicted in Fig. 5 which is mostly caused by morphology changes of very thin metal films deposited on a low refractive index fluoropolymer (e.g., Teflon or Cytop with low surface energy are used to generate the symmetrical refractive index structure) [61]. As the probing depth $L_{\mathrm{p}}$ of LRSPPs can reach up to several microns, it allows for order of magnitude higher fluorescence signals for architectures where fluorophores are dispersed in an extended 3D matrix rather than attached on a surface or embedded in a thin dielectric film [60].

As Fig. 2a shows, SPPs can efficiently collect fluorescence light (more than $50 \%$ photons) emitted at emission wavelength $\lambda_{\mathrm{em}}$ from a close proximity to a metallic surface. Figures $1 \mathrm{a}$ and $3 \mathrm{a}$ illustrate that the surface plasmon-coupled fluorescence emission (SPCE) can tunnel through a thin metal film into a dielectric substrate where emitted light forms a highly directional characteristic cone propagating into the far field. This type of emission at $\lambda_{\mathrm{em}}$ can be combined with the excitation via SPPs at $\lambda_{\mathrm{ab}}$ which occurs at a slightly different angle [62]. In order to collect the SPCE signal that is isotropic in azimuthal angle $\phi$, elements such as hemispherical prism [62], dielectric paraboloid element [63], and concentric diffraction grating [64] were developed (see Fig. 6). The use of LRSPPs to collect fluorescence light is less efficient than regular SPPs (owing to the weaker field confinement) but offers the advantage of narrower angular distribution and higher peak intensity of SPCE [65]. In addition, let us note that SCPE can be canceled by the design of SPP dispersion relation, so a bandgap occurs at wavelengths close to $\lambda_{\mathrm{em}}$ [66].

\section{Periodically Corrugated Continuous Metallic Films}

Diffraction on periodically corrugated metallic surfaces provides an alternative means for simultaneous SPP-enhanced 
Table 2 Experimentally determined fluorescence enhancement factors EF with the information on plasmonic nanostructure, type of supported surface plasmon modes, and intrinsic quantum yield of used fluorophore.
Studies in which dyes were attached at the distance $d=10-20 \mathrm{~nm}$ from a metal are preferably selected

\begin{tabular}{|c|c|c|c|c|}
\hline Plasmonics structure & $\mathrm{EF}$ & Fluorophore $\left(\eta_{0}\right)$ & $f$ & Ref. \\
\hline \multicolumn{5}{|l|}{ Continuous metallic films } \\
\hline Au, ATR-coupled SPP & 32 & Rhodamine-6G (0.95) & High & {$[58]$} \\
\hline Au, ATR-coupled SPP & 17 & MR 21 & & [104] \\
\hline $\mathrm{Ag}, 2 \mathrm{D}$ grating-coupled SPP & 100 & Cy5 (0.28) & High & {$[68]$} \\
\hline $\mathrm{Au}, 1 \mathrm{D}$ grating-coupled SPP & 13 & CdSe-ZnS QD (0.45) & High & [122] \\
\hline $\mathrm{Au}, 1 \mathrm{D}$ grating-coupled SPP & 24 & Cy5 (0.28) & High & [123] \\
\hline Au bull's eye, hybrid SPP and LSP & 77 & AF $647(0.3)$ & High & {$[34]$} \\
\hline $\mathrm{Ag}, 1 \mathrm{D}$ grating SPP & 30 & Rhodamine 6G (0.95) & High & [124] \\
\hline \multicolumn{5}{|l|}{ Nanoclusters } \\
\hline $\mathrm{Ag} / \mathrm{Au}, 2 \mathrm{D}$ nanoclusters, LSP & 9.4 & Cy5 (0.28) & & {$[125]$} \\
\hline $\mathrm{Ag} / \mathrm{Au}, 2 \mathrm{D}$ nanoclusters, LSP & 35 & Cy5 (0.28) & & {$[72]$} \\
\hline Ag, rough nanopourous film, LSP & 30 & Rhodamine-6G (0.95) & & [126] \\
\hline $\mathrm{Ag}$, island film, LSP & 50 & Bis benzimidazo perylene & & [127] \\
\hline \multicolumn{5}{|l|}{ Chemically synthesized nanoparticles } \\
\hline Au core dielectric shell, LSP & 40 & $\operatorname{IR} 800(0.07)$ & & [73] \\
\hline Ag spherical NP, LSP & $13-15$ & AF488 (0.92) & & {$[14]$} \\
\hline Au spherical NP, LSP & $8-10$ & Nile Blue (0.8) & & [128] \\
\hline Ag spherical NP aggregated, LSP & 170 & Atto $655(0.13)$ & & {$[75]$} \\
\hline Ag spherical NP on Ag film, SPP coupled with LSP & 1,000 & perylene diimide & High & {$[76]$} \\
\hline Au nanorod, LSP & 20.8 & Oxazine-725 & & [74] \\
\hline Ag spherical hollow NP, LSP & 300 & Cy5 (0.28) & & [129] \\
\hline Ag core dielectric shell, LSP & 94 & Octadecyl Rhodamine B (R18) & & [130] \\
\hline \multicolumn{5}{|l|}{ Lithography fabricated nanoparticles } \\
\hline Bow tie NP, LSP & 1,340 & TPQDI $(0.025)$ & & [43] \\
\hline Au gap-antenna, LSP & 1,100 & Alexa Fluor 647 with quencher (0.08) & & {$[57]$} \\
\hline $\mathrm{Au}$ D2PA, LSP & 2,970 & ICG (0.012) & & {$[78]$} \\
\hline Ag nanodisks, LSP & 15 & Cy3 (0.04) & & [79] \\
\hline Au nanodisks, LSP & 15 & CdSe-ZnS QDs (0.3-0.5) & & {$[80]$} \\
\hline Au nanoholes, hybrid SPP and LSP & 82 & Oxazine $720(0.6)$ & & {$[82]$} \\
\hline Ag nanoholes, hybrid SPP and LSP & 110 & Cy5 (0.28) & & {$[68]$} \\
\hline Au nanodisk over metal film, LSP & 600 & $\operatorname{IR} 800(0.07)$ & & [131] \\
\hline Au nanotriangle, LSP & 83 & Alexa Fluor 790 (0.04) & & {$[48]$} \\
\hline
\end{tabular}

excitation at $\lambda_{\mathrm{ab}}$ and extraction of SPP-driven emission of fluorescence light at $\lambda_{\text {em }}[55,67]$. For example, this combined approach allowed for the enhancement of fluorescence signal with a factor of $E F=40$ and $10^{2}$ for $1 \mathrm{D}$ and crossed $2 \mathrm{D}$ gratings, respectively [68]. These results were obtained for a medium quantum yield Cy5 dye immobilized on a gold grating with the modulation period of $\Lambda=400 \mathrm{~nm}$, depth of 20 $25 \mathrm{~nm}$, and a 20-nm-thick $\mathrm{SiO}_{2}$ spacer layer preventing quenching [68]. A metallic circular grating (so-called bull's eye) with a nanohole in its center was employed for the amplification of fluorescence signal emitted by dyes that diffused in the nanohole cavity [34, 69] (see the respective figure in Table 1). Compared to regular gratings, a larger enhancement factor of $\mathrm{EF}=1.2 \times 10^{2}$ was reported for medium quantum yield Alexa Fluor $647\left(\eta_{0}=0.33, \lambda_{\mathrm{ab}} \sim 650 \mathrm{~nm}\right.$, and $\left.\lambda_{\mathrm{em}} \sim 665 \mathrm{~nm}\right)$ and reference flat gold film structure [34]. This amplification strategy took advantage of surface plasmon coupling at both $\lambda_{\mathrm{ab}}$ and $\lambda_{\mathrm{em}}$ wavelengths. Figure 7 illustrates how the design of periodic concentric grating allowed controlling the directionality of SPP-driven fluorescence emission by changing the phase of SPP modes that scattered on concentric grooves.

Metallic Islands and Nanoclusters

Fluorescence enhancement on substrates with metallic islands and nanoclusters was subject to research since the 1980s [70]. This approach offers the advantage of a relatively simple 
Fig. 6 Optical elements for the extraction of SPCE utilizing reverse Kretschmann configuration and a paraboloid elements and $\mathbf{b}$ concentric diffractive lens (reproduced with permission from [63] and [66])

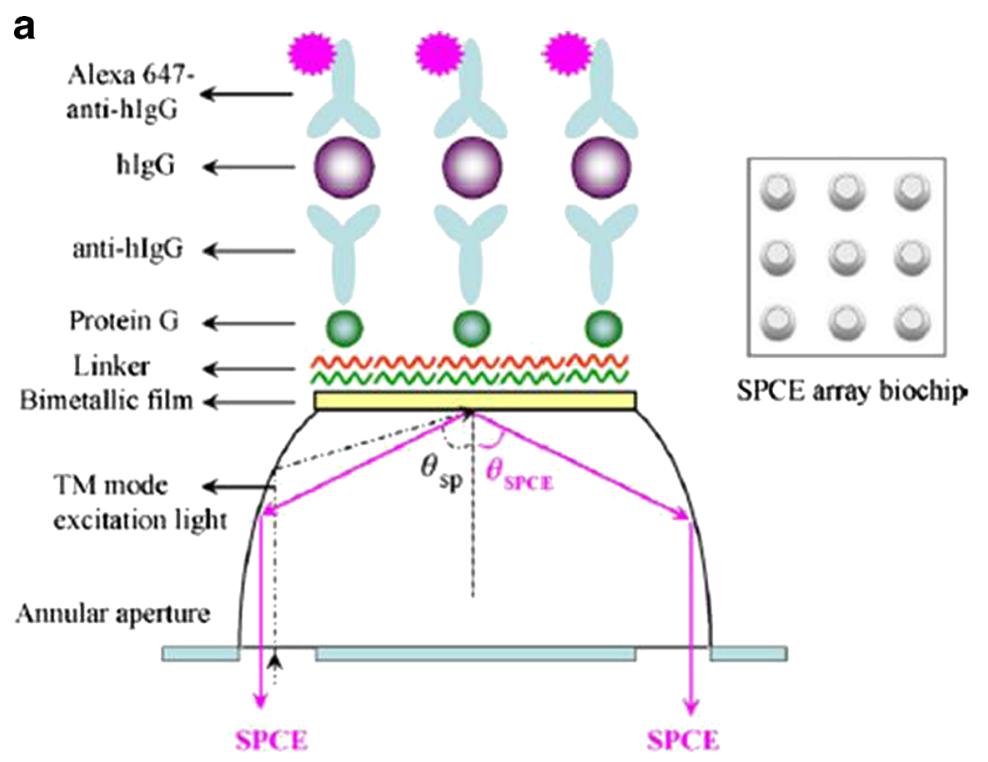

b

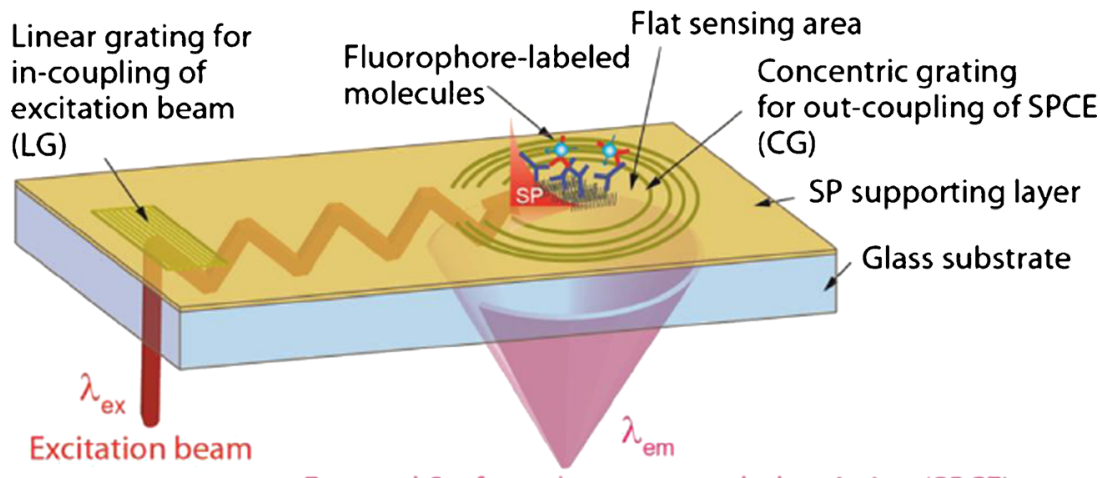

Focused Surface plasmon-coupled emission (SPCE) preparation procedure and provides moderate enhancement factors through the combined effect of LSP field-enhanced fluorescence excitation rate $\gamma_{\mathrm{e}}$ and increased quantum yield $\eta$. For instance, silver islands with size between 20 and $80 \mathrm{~nm}$ enhanced the fluorescence signal from adsorbed bovine serum albumin protein that was conjugated with Texas Red dye $\left(\eta_{0}=\right.$ $0.2, \lambda_{\mathrm{ab}}=590 \mathrm{~nm}$, and $\lambda_{\mathrm{em}}=615 \mathrm{~nm}$ ) by a factor of $\mathrm{EF}=8-16$
[71]. These structures exhibited a broad LSPR absorption band centered at a wavelength of $\lambda \sim 450 \mathrm{~nm}$ that was below the dye excitation and emission bands. Annealing a thin stack of silver and gold films with varied thicknesses allowed for tuning LSPR wavelength of bi-metal nanoclusters between $\lambda=450$ and $550 \mathrm{~nm}$ [72]. These structures were coated with an amorphous silicon-carbon alloy which simultaneously served
Fig. 7 Diffraction control of angular distribution of fluorescence emission by series of concentric grooves (bull's eye structure presented in Table 1) with varied offset $a$ between the first groove and the aperture center (reproduced with permission from [69])
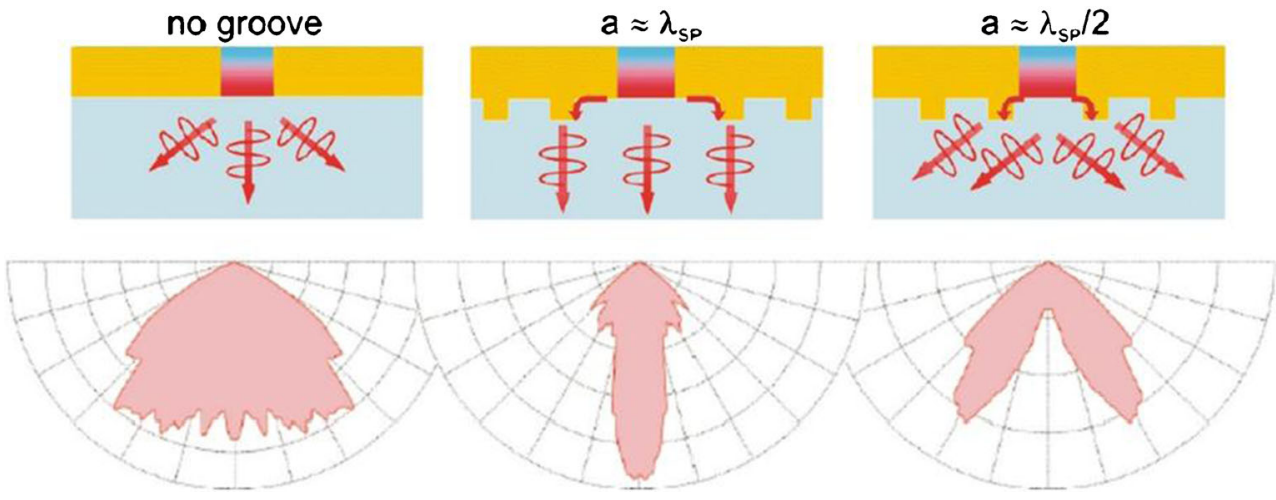
as a protection and spacer layer. Obtained results showed that the enhancement increases when the LSPR wavelength is tuned towards $\lambda_{\mathrm{ab}}$ and $\lambda_{\mathrm{em}}$ of used Cy5 dye, and the maximum value of $\mathrm{EF}=35$ was achieved.

\section{Chemically Synthesized Metallic Nanoparticles}

A chemically synthesized spherical metallic nanoparticle was attached to a sharp glass tip and it was approached individual dyes on a glass substrate [14]. This arrangement allowed for precise control of the distance $d$ between the nanoparticle and fluorophore. Obtained results revealed an optimum distance of around $d \sim 10 \mathrm{~nm}$ for high quantum yield Alexa Fluor 488 dye $\left(\eta_{0}=0.92, \lambda_{\mathrm{ab}} \sim 495 \mathrm{~nm}, \lambda_{\mathrm{em}} \sim 519 \mathrm{~nm}\right)$ and silver nanoparticle with a diameter of $80 \mathrm{~nm}$. At this distance, the fluorescence intensity emitted into the glass substrate was enhanced by a factor of $E F=13-15$ when the dye was excited via LSPs at the wavelength of $488 \mathrm{~nm}$ [14]. The same work reported similar enhancement of $\mathrm{EF}=8-9$ for medium quantum yield Nile Blue dye $\left(\eta_{0}=0.27, \lambda_{\mathrm{ab}} \sim 627 \mathrm{~nm}, \lambda_{\mathrm{em}}\right.$ $\sim 630 \mathrm{~nm}$ ) and gold spherical nanoparticle supporting LSPR at longer wavelength of $\lambda=637 \mathrm{~nm}$. Gold nanoshell particles can be used for the fluorescence enhancement in near infrared (NIR) spectrum. Nanoshell particles with $15 \mathrm{~nm}$ thick gold capping layer, outer diameter of $78 \mathrm{~nm}$, and LSPR wavelength $\lambda \sim 800 \mathrm{~nm}$ were decorated with human serum albumin conjugated with low quantum yield IR800 dye $\left(\eta_{0}=0.07, \lambda_{\mathrm{ab}}=\right.$ $745 \mathrm{~nm}, \lambda_{\mathrm{em}}=795 \mathrm{~nm}$ ) [73]. Measured fluorescence intensity (emitted per attached dye) was enhanced by a factor of $E F=40$ with respect to that for an identical labeled protein in a solution. The same study showed that a gold rod nanoparticle with LSPR wavelength at $\lambda \sim 800 \mathrm{~nm}$ enhanced the fluorescence signal by a lower factor of $\mathrm{EF}=9$. This enhancement was increased when the transversal and longitudinal LSPRs were engineered to spectrally overlap with fluorophore absorption $\lambda_{\mathrm{ab}}$ and emission $\lambda_{\mathrm{em}}$ wavelengths. The enhancement of $E F=20.8$ was obtained for Oxazine-725 dye on gold rod nanoparticles with transverse and parallel LSP modes tuned to wavelengths 532 and $720 \mathrm{~nm}$, respectively [74]. Significantly stronger enhancement of $\mathrm{EF}=1.7 \times 10^{2}$ was observed for fluorophore molecules exposed to more tightly confined field in gaps between plasmonic nanoparticles [75]. This approach was studied by using aggregates of spherical silver nanoparticles with a diameter of $37 \mathrm{~nm}$ and trapped medium quantum yield Atto-655 dyes $\left(\eta_{0}=0.3, \lambda_{\mathrm{ab}}=663 \mathrm{~nm}, \lambda_{\mathrm{em}}=684 \mathrm{~nm}\right)$. Even larger enhancement factor of EF $\sim 1.1 \times 10^{3}$ was reported for perylene diimide dye that was dispersed in a 2-3-nm spacer layer between a silver naoparticle (diameter of $80 \mathrm{~nm}$ ) and a flat silver surface supporting a confined gap LSP mode [76]. However, it should be noted that such large EF value was partially obtained due to the fact that the reference measurement was performed for a dye at a very small distance of $d=2-$
$3 \mathrm{~nm}$ from a silver surface (which leads to strong quenching; see Fig. 2b).

\section{Metallic Nanostructure Arrays Prepared by Lithography}

Modern lithography provides powerful fabrication tools for the preparation of metallic nanostructures that can be tailored for very efficient PEF studies on individual fluorophore molecules. Fluorescence enhancement of $\mathrm{EF}=1.3 \times 10^{3}$ was reported for bow tie nanoparticles (see the respective figure in Table 1$)$ and a low quantum yield TPQDI dye $\left(\eta_{0}=0.025, \lambda_{\mathrm{ab}}\right.$ $\sim 790 \mathrm{~nm}, \lambda_{\mathrm{em}}=850 \mathrm{~nm}$ ) [43]. These structures were prepared by electron beam lithography (EBL), and it is important to note that such high EF was observed for individual molecules that were positioned in an approximately 30 -nm-wide gap between the sharp nanoparticle tips. A similar enhancement factor of $\mathrm{EF}=1.1 \times 10^{3}$ was obtained for low quantum yield Alexa Fluor dye (reduced to $\eta_{0}=0.08$ by a quencher) two half cylindrical nanoparticles fabricated by focused ion beam milling (FIB) [57]. Even more complex metallic nanoparticle geometries such as those resembling Yagi Uda nanoantenna were prepared by EBL for the fluorescence measurements on single emitters including quantum dots [77] or dyes [18]. Nanoimprint lithography was used for the preparation of dense arrays hierarchical structures comprising gold disk nanoparticles with around $100 \mathrm{~nm}$ diameter above a metallic backplane. Inside the narrow gap between disk nanoparticles and the backplane, additional gold clusters with a diameter of 5-25 $\mathrm{nm}$ were formed [78]. This system exhibited broad LSPR resonance centered at around $800 \mathrm{~nm}$, and it was reported to allow for large EF of fluorescence light emitted from low quantum yield infrared dye indocyanine green (ICG, $\left.\eta_{0}=0.012, \lambda_{\mathrm{ab}}=783 \mathrm{~nm}, \lambda_{\mathrm{em}} \sim 850 \mathrm{~nm}\right)$. The enhancement factor of $1.1 \times 10^{3}$ was measured for an ensemble of dyes that were randomly attached at the distance of $d=5 \mathrm{~nm}$ from the gold surface. In addition, experiments on individual dyes indicated an enormous maximum enhancement of $\mathrm{EF}=4.5 \times 10^{6}$.

Periodic arrays of metallic nanoparticles with weakly interacting dipole LSPs were used in numerous investigations with ensembles of fluorophores. Rectangular arrays of silver disk nanoparticles with a diameter of $120 \mathrm{~nm}$ and height of $27 \mathrm{~nm}$ were prepared by nanoimprint lithography (NIL) and showed the maximum enhancement factor of $\mathrm{EF}=15.8$ per attached low quantum yield Cy 3 dye $\left(\eta_{0}=0.04, \lambda_{\mathrm{ab}}=550 \mathrm{~nm}\right.$, and $\lambda_{\mathrm{em}}=570 \mathrm{mn}$ ) [79]. The period of the structure was adjusted to $\Lambda=200 \mathrm{~nm}$ in order to match the LSPR wavelength to that of focused excitation beam $(\lambda=543 \mathrm{~nm})$ and $\mathrm{Cy} 3$ dye absorption wavelength $\lambda_{\mathrm{ab}}$. Similar fluorescence enhancement was reported for arrays of gold nanodisk and $\mathrm{CdSe}-\mathrm{ZnS}$ quantum dots $\left(\lambda_{\mathrm{em}} \sim 600 \mathrm{~nm}\right.$ ) which were excited by a broad wavelength spectrum of mercury lamp [80]. Quantum dots were selectively attached to the gold nanoparticles with varied thickness of the spacer film that was prepared by successive 
coating by 1-4-dibiotinylbutane and streptavidin layers. The highest (area compensated) enhancement was achieved for the structure with spacer layer thickness of $d=16 \mathrm{~nm}$, disk diameter of $100 \mathrm{~nm}$, and period of $\Lambda=200 \mathrm{~nm}$ which supported LSPs at $\lambda=580 \mathrm{~nm}$. Dense arrays of silver nanotriangles which were produced by colloidal lithography revealed fluorescence amplification of $\mathrm{EF}=83$ for Alexa Fluor $790\left(\eta_{0}=\right.$ $0.04, \lambda_{\mathrm{ab}}=782 \mathrm{~nm}$, and $\lambda_{\mathrm{em}}=804 \mathrm{mn}$ ) [48].

Complementary structures with metallic films perforated by nanohole arrays were utilized for PEF that takes advantage of the interplay between SPP and nanohole LSP modes. For instance, EBL-fabricated nanohole arrays with a diameter of $100 \mathrm{~nm}$ and period between $\Lambda=350$ and $650 \mathrm{~nm}$ were reported to enhance the fluorescence signal by a factor of $E F=82$ for Oxazine $720\left(\eta_{0}=0.6, \lambda_{\mathrm{ab}}=620 \mathrm{~nm}, \lambda_{\mathrm{em}}=650 \mathrm{~nm}\right.$ [81] $)$ and the excitation wavelength $\lambda=633 \mathrm{~nm}$ [82]. This enhancement was achieved for a period of $\Lambda=553 \mathrm{~nm}$ which allowed simultaneous excitation and emission of fluorescence light by LSPs supported by nanoholes and diffraction-coupled SPPs. Similar enhancements of $\mathrm{EF}=1.1 \times 10^{2}$ have been reported for $\mathrm{Cy} 5$ on a silver nanohole array protected by a 20 $\mathrm{nm} \mathrm{SiO}_{2}$ spacer film [68].

In summary, the coupling of fluorophores with intense fields of surface plasmons can amplify emitted fluorescence light intensity by several orders of magnitude. The highest enhancements were demonstrated in measurements with single fluorophores that were placed into plasmonic hotspots. In combination with low quantum yield dyes, several groups reported the fluorescence enhancement $>10^{3}$ for such configurations.

\section{Interface Architectures}

In order to exploit the amplification of fluorescence signal in detection assays, surfaces of metallic nanostructures have to carry biomolecular recognition elements (BREs) that can specifically capture the target analyte from a liquid sample. As such surface chemistries were already subject to thorough reviews [83, 84], this section provides only a brief overview of commonly used building blocks. Rather, we focus on biointerfacial systems that were adopted for selective (local) attachment of biomolecules at plasmonic hotspots. We discuss some key implications for the sensitivity of fluorescence biosensors which utilize such structures. In particular, it is important to note that the local functionalization of plasmonic hotspots is on the one hand favorable as it assures high fluorescence signal associated with a binding event, but on the other hand it leads to lower average density of BREs on the sensor surface and potentially to smaller probability of analyte capture. These two effects may act counter each other and thus hinder the sensitivity of PEF biosensor technologies.
Functionalization Building Blocks

In biosensor applications, self-assembled monolayers (SAMs) represent a popular class of materials used for tailoring properties of interfaces between a transducer and liquid sample [85-87]. Alkanethiol SAMs offer a powerful toolbox for reliable attachment of biomolecules to noble metal surfaces via amine coupling, his-tag, and biotin-streptavidin interaction. Silanes-based chemistries are preferably used for the functionalization of oxide layers $[72,88]$ which often serve as a protection layer and a spacer film for the control of a distance between a fluorophore and metal $d$. S-layer protein SAMs were employed for the modification of surfaces of plasmonic biosensors, and specific fusion proteins carrying functional groups that react with biotin tags [89] or immunoglobulin (IgG) Fc regions $[90,91]$ were developed. Another important route for the functionalization of metallic surfaces utilizes synthetic or natural polymers. When attached to the metal surface, they can provide an open 3D structure that accommodates larger amounts of biomolecules than a 2D system relying on SAMs. For instance, poly( $N$-isopropylacryamide) [61] and dextranbased [92] cross-linked polymer networks and dextran-based brushes [93] were successfully utilized in PEF biosensors that took advantage of high-binding capacity matrices (see Fig. 8a). In order to control the distance between fluorophores and a metallic surface $d$, layer-by-layer deposition of polymer spacer layers was commonly used $[4,94]$.

\section{Local Functionalization}

Precise attachment of BREs to areas where electromagnetic field is confined (plasmonic hotspots) is crucial in order to harness the large fluorescence signal amplification enabled by PEF on metallic nanostructures. The reason is that only those molecular binding events occurring in plasmonic hotspots contribute to a strongly amplified fluorescence signal while the binding taking place outside plasmonic hotspots does not. EBL was proposed for the selective functionalization of gold nanorod arrays by using a PMMA mask with clearance windows for selective access to nanorod tips [95]. Another potentially simpler approach based on material selective surface modification was reported for arrays of metallic nanoholes [96]. In this work, colloidal lithography was used to etch nanoholes through a stack of $\mathrm{TiO}_{2}-\mathrm{Au}-\mathrm{TiO}_{2}$ films (see Fig. 8b). The gold nanohole walls were modified with thiol-PEG carrying a biotin terminal group while the $\mathrm{TiO}_{2}$ oxide surface was passivated by poly(Llysine)-graft-PEG (PLL-g-PEG). On this structure, selective binding of neutravidin to the gold nanohole walls was observed with LSPR. Near-field lithography was suggested for the selective attachment of molecules close to plasmonic nanoparticle hotspots by using a polysiloxane layer containing a nitroveratrylcarbonyl (NVoc) [97]. The excitation of LSPs at the wavelength $780 \mathrm{~nm}$ by a pulsed laser beam locally 
Fig. 8 Example of a threedimensional binding capacity binding matrix utilizing a crosslinked polymer network (reproduced with permission from [92]) and b local modification of inner walls of cylindrical metallic nanoholes with two-dimensional SAM by using material-selective local chemistries (reproduced with permission from [96])

\section{a}
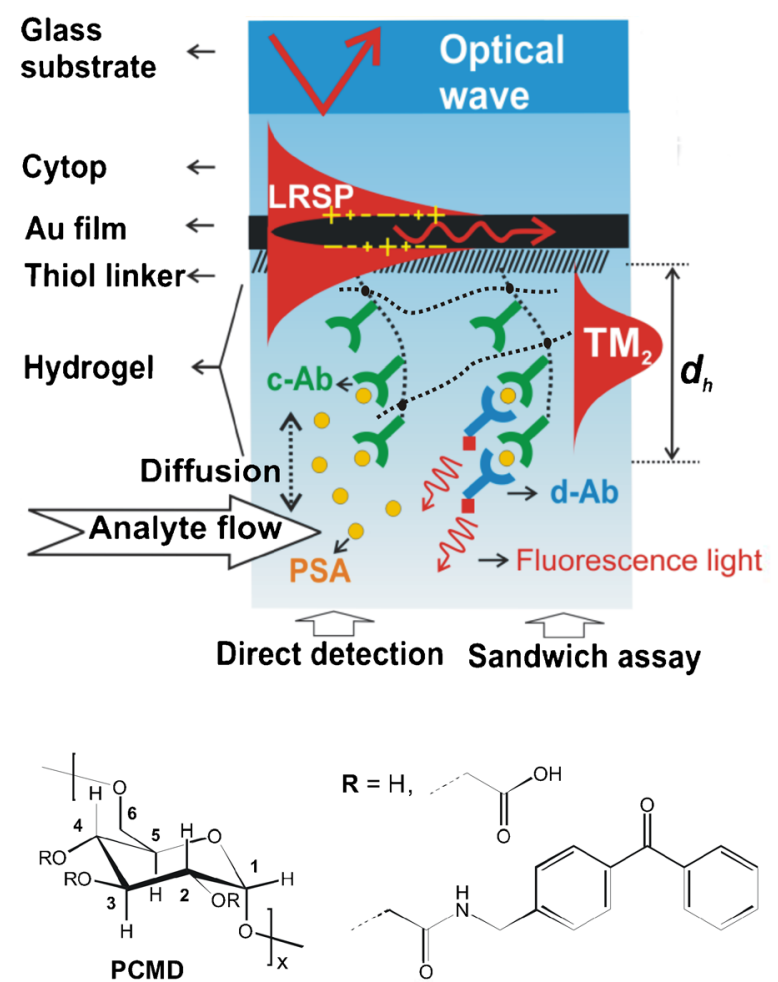

b
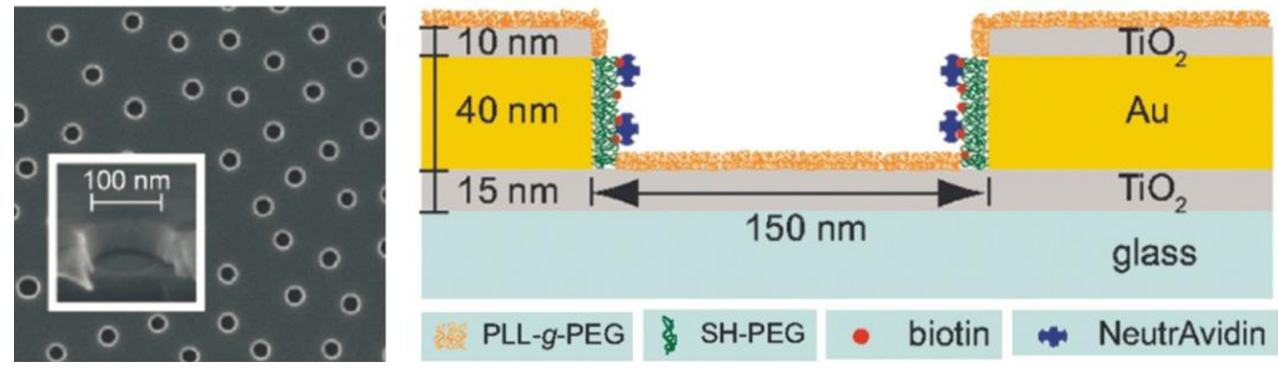

amplified two-photon absorption of NVoc which leads to its cleavage. This approach was envisaged to open new ways for preparing nanoscale windows around the metallic particles for subsequent selective modification with proteins or synthetic functional polymers. Selective functionalization of gold nanorods prepared by wet chemical synthesis is possible due to the high crystallinity of such nanoparticles [98]. For instance, cetyltrimethylammonium bromide (CTAB) that is used for the stabilizing of gold nanorod particles preferably bind to $\{100\}$ faces of nanorods leaving the $\{111\}$ nanorod tips available for the attachment of other moieties such as biotin disulfide. A similar approach was employed in PEF studies for the covalent linkage of fluorophores at the preferred longitudinal axis of the gold nanorods [99].

\section{Affinity Binding at Plasmonic Hotspots}

In biosensors, measured sensor signal is calibrated against the concentration of target analyte in an analyzed sample $c_{\alpha}$. For fluorescence-based heterogeneous assays, measured fluorescence signal $F$ is proportional to the product of the enhancement factor EF and number of specifically captured molecules on a sensing spot. The relation between the number of captured molecules and the concentration of analyte in a sample $c_{\alpha}$ depends on range of parameters including the means of the analyte transfer from a sample to the surface, density of biomolecular recognition elements $c_{\beta}$, dissociation affinity binding constant $K_{\mathrm{d}}$, and reaction time. By using the Langmuir isotherm, one can show that the fluorescence signal can be described by the following equation:

$$
F \propto \mathrm{EF} \cdot S \frac{c_{\alpha}}{\frac{K_{\mathrm{d}}}{\xi c_{\beta}}+\frac{S}{V}}
$$

This equation holds for the analyte concentrations much smaller than dissociation constant $c_{\alpha} \ll K_{\mathrm{d}}$ and surface reaction in equilibrium. $V$ denotes the volume of analyzed sample with an analyte concentration of $c_{\alpha}, S$ is the surface area of a 
sensing spot, and $\xi$ is the fraction of this area that is occupied by plasmonic hotspots and functionalized by BREs with surface density of $c_{\beta}$. For large sample volumes $V$ and small functionalized surface areas $S$, the sensor response is proportional to term $\sim \mathrm{EF} \xi S c_{\beta} c_{\alpha}$. In this limit, the effect of strong PEF amplification at sparsely distributed hotspots will not provide substantially improved sensitivity. The reason is that large EF factors are typically associated with strong electromagnetic field confinement which occurs for a low density of hotspots $\xi$. However, Eq. (5) indicates that PEF on locally functionalized hotspot would be highly favorable for the analysis of small sample volumes $V$ when high affinity BREs are used. For $c_{\beta} S \gg K_{d} V$, Eq. (5) yields $F \propto E F V c_{\alpha}$ which translates to the situation when virtually all present molecules are captured at plasmonic hotspots and contribute to amplified fluorescence signal. For example, let us assume that IgG antibodies serve as biomolecular recognition elements and they are immobilized on the hotspot surface with the full packed monolayer density of $c_{\beta} \sim 2.5 \times 10^{-14} \mathrm{~mol} / \mathrm{mm}^{2}$. The sensing spot area is $S=1 \mathrm{~mm}^{2}$, and plasmonic hotspots occupy its $10 \%(\xi=0.1)$. Then, the above condition is fulfilled when that sample volume of $10 \mu \mathrm{L}$ is analyzed and dissociation constant of BREs is better than $K_{\mathrm{d}}=0.25 \times 10^{-9} \mathrm{M}$. Even most of the used antibodies exhibit the $K_{\mathrm{d}}$ in the nanomolar range, numerous antibodies with $K_{\mathrm{d}}$ as low as $10^{-12} \mathrm{M}$ become available $[100,101]$ which opens room for highly sensitive immunoassay detection schemes with spatially confined plasmonic hotspots. It should be noted that another important parameter is the time needed for the collecting of the analyte on a surface. In order to speed up this process, PEF biosensors can be combined with ultrasound sonication [102], microwave heating [103], or they rely microfluidic devices [104].

\section{Biosensor Applications}

Over the last years, we witnessed numerous implementations of PEF into already established laboratory technologies such as fluorescence microarray scanners, fluorescence microscopes, or microtiter plate readers as well as the development of entirely new compact devices that utilize this amplification scheme. PEF nanostructures were mostly combined with immunoassays which offer the advantage of commercial availability of antibodies against a large variety of analytes (see Table 3). In order to avoid direct labeling of target analyte, there are typically used sandwich $[105,106]$ or competitive [105] assay formats with detection antibody conjugated to a fluorophore (see Fig. 8a).

The first biosensor implementation of PEF was reported in the beginning of the 1990s [58], and a decade later, it was

Table 3 Overview of PEF biosensors for the detection of chemical and biological compounds with information on analyzed matrix, limit of detection, analysis time, and assay format

\begin{tabular}{|c|c|c|c|c|c|c|}
\hline Analyte & Plasmon mode & Matrix & Limit of detection & Detection time & Assay type & Ref. \\
\hline \multicolumn{7}{|l|}{ Model analytes } \\
\hline DNA & SPP & Buffer & $30 \mathrm{pM}$ & $10 \min$ & Direct & [132] \\
\hline DNA & SPP & Buffer & $1.57 \mathrm{pM}$ & $30 \mathrm{~min}$ & Direct & [89] \\
\hline RNA & LSP & Buffer & $25 \mathrm{fM}$ & $30 \mathrm{~min}$ & Sandwich & [133] \\
\hline a-mouse $\operatorname{IgG}$ & LRSPP & Buffer & $20 \mathrm{fM}$ & $20 \mathrm{~min}$ & Direct & {$[61]$} \\
\hline Streptavidin & SPP & Buffer & $50 \mathrm{pM}$ & $10 \mathrm{~min}$ & Direct & {$[88]$} \\
\hline Human IgG & SPP & Buffer & $1 \mathrm{pg} / \mathrm{ml}(6.7 \mathrm{fM})$ & $1 \mathrm{~h}$ & Sandwich & [106] \\
\hline Human IgG & LSP & Buffer & $86 \mathrm{pg} / \mathrm{ml}(0.57 \mathrm{pM})$ & $1 \mathrm{~h}$ & Sandwich & [116] \\
\hline Human IgG & LSP & Buffer & $0.3 \mathrm{fM}$ & $1 \mathrm{~h}$ & Direct & [107] \\
\hline \multicolumn{7}{|l|}{ Biomarkers } \\
\hline f-PSA & LRSPP & Buffer/serum & $34 / 330 \mathrm{fM}$ & $35 \mathrm{~min}$ & Sandwich & [92] \\
\hline Total PSA & LSP & Buffer/serum & $0.4 / 1.8 \mathrm{pg} / \mathrm{ml}(12 / 52 \mathrm{fM})$ & $1 \mathrm{~h}$ & Sandwich & [111] \\
\hline TNF- $\alpha$ & LSP & n.a. & $3 \mathrm{pM}$ & $2 \mathrm{~h}$ & Sandwich & [114] \\
\hline Troponin I & LSP & Buffer/blood & $5 / 50 \mathrm{pg} / \mathrm{ml}(0.22 / 4.3 \mathrm{pM})$ & $1 \mathrm{~min}$ & Sandwich & [115] \\
\hline C-reactive protein & SPP & Buffer/serum & $16 / 26 \mathrm{ng} / \mathrm{ml}(0.15 / 0.25 \mathrm{nM})$ & $30 \mathrm{~min}$ & Sandwich & [112] \\
\hline UL16-binding protein 2 & LSP & Serum & $18 \mathrm{pg} / \mathrm{ml}(0.75 \mathrm{pM})$ & $4.3 \mathrm{~h}$ & Sandwich & [113] \\
\hline \multicolumn{7}{|l|}{ Pathogens and toxins } \\
\hline Aflatoxin $\mathrm{M}_{1}$ & LRSPP & Buffer/milk & $0.6 \mathrm{pg} / \mathrm{ml}(1.8 \mathrm{pM})$ & $53 \mathrm{~min}$ & Inhibition & [105] \\
\hline E. coli 157 & LRSPP & Buffer & $6 \mathrm{cfu} / \mathrm{ml}$ & $20 \mathrm{~min}$ & Sandwich & [119] \\
\hline SARS-CoV & LSP & Buffer & $13.9 \mathrm{pg} / \mathrm{ml}$ & n.a. & Sandwich & [120] \\
\hline S-OIV & LSP & Buffer/serum & $0.1 / 1 \mathrm{pg} / \mathrm{ml}$ & n.a. & Sandwich & [121] \\
\hline Anthrax protective antigen & LSP & Buffer & $0.1 \mathrm{pg} / \mathrm{ml}$ & $40 \mathrm{~min}$ & Inhibition & [134] \\
\hline
\end{tabular}


reintroduced in the form of a method named surface plasmonenhanced fluorescence spectroscopy (SPFS) [104]. This approach takes advantage of the enhancement of fluorescence signal via probing the metal sensor surface with SPPs that are resonantly excited at the absorption wavelength $\lambda_{\mathrm{ab}}$ of used fluorophore labels. Another configuration utilizing SPPs for the collecting of fluorescence light at the fluorophore emission wavelength $\lambda_{\mathrm{em}}$ was developed based on surface plasmoncoupled emission (SPCE) [62]. The most common implementations of SPFS method utilize an optical setup with angular interrogation of SPR and an additional module for the collecting and detection of emitted fluorescence intensity (see Fig. 9a). In this scheme, analyzed samples are flowed over the sensor chip with SPR-active layer modified by biomolecular interaction elements. The capture of the target analyte occurring on the sensor surface can be observed by combined SPR and measurement of intensity of fluorescence light that is emitted through a sample above the metal surface (see Fig. 9b). Both SPR and fluorescence signals can be monitored

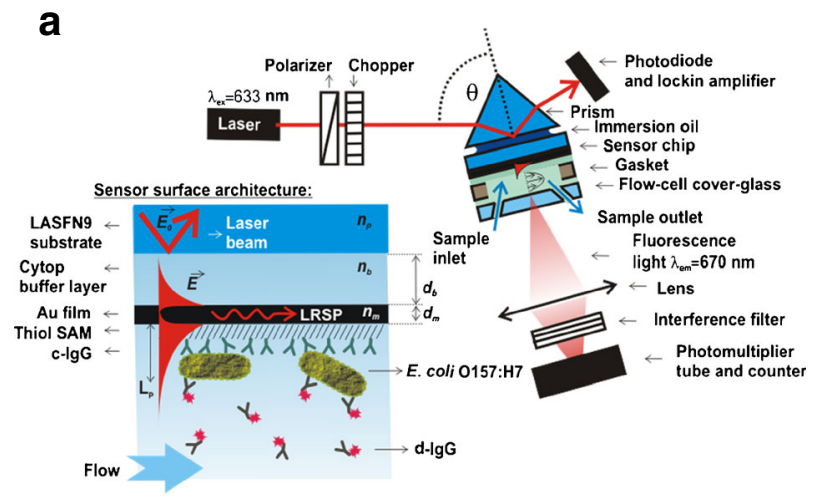

b

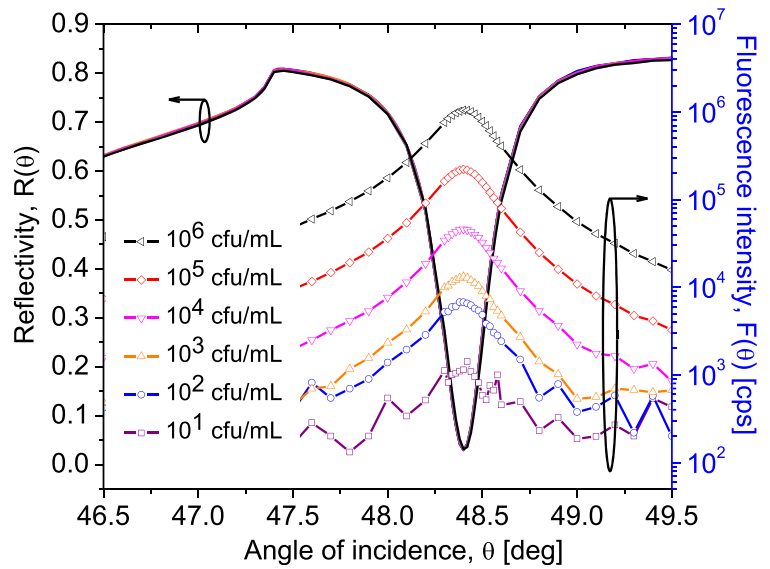

Fig. 9 a Optical setup of surface plasmon-enhanced fluorescence spectroscopy (SPFS) utilizing angular modulation of SPR. Example of a sensor chip supporting LRSPPs and E. coli O157:H7 sandwich immunoassay format. b Fluorescence signal measured upon the changing angle of incidence of the excitation laser beam in vicinity to the resonance after binding of target analyte (E. coli $\mathrm{O} 157: \mathrm{H} 7)$ and reacting with dye-labeled detection antibody on the surface (reproduced with permission from [119]) in real time which allowed for advanced biomolecular interaction analysis (BIA) studies [88, 93, 104]. SPFS was shown to detect molecular analytes such as immunoglobulin $\mathrm{G}(\mathrm{IgG})$ at a concentration as low as $0.5 \mathrm{fM}$ [4]. SPCE is implemented by using a similar Kretschmann configuration as SPFS, but the intensity of fluorescence light that is emitted into a substrate below the metal film is measured. The SPCE detection format with a disposable biochip carrying arrays of embossed paraboloid elements was reported [106] (see Fig. 6). By utilizing SPP-driven excitation and emission of fluorescence light on a thin metallic deposited on top of such elements, IgG assay with limit of detection as low as $1 \mathrm{pg} / \mathrm{ml}(6 \mathrm{fM})$ was demonstrated.

Diffraction gratings supporting SPPs [88] and substrates with metallic nanoparticles exhibiting LSPR [72] were applied for the amplified fluorescence measurements performed by commercially available fluorescence microscopy and microarray scanners. Typically, an end-point fluorescence signal is measured after the reaction of the analyte with BREs on the surface. In conjunction with commercially available fluorescence scanners, limits of detection between femtomolar and picomolar concentrations were most often reported $[72,88]$. So far, the best limit of detection of $0.3 \mathrm{fM}$ was achieved for direct detection of IRDye-800cw dye-labeled IgG molecules and a dense grating combining NIL-prepared metallic gaps and random metallic clusters [107]. In general, sensor chips with metallic nanostructures that can be fabricated by mass production-compatible technologies (such as colloidal lithography, NIL, annealing of thin films, or wet chemical synthesis) are better suited for practical PEF biosensors than techniques that require slow and expensive nanofabrication tools (EBL or FIB).

\section{Detection of Biomarkers}

Prostate-specific antigen (PSA) is an established biomarker for the diagnosis of prostate cancer and new technologies for its analysis at concentrations below picomolars are expected to provide a valuable tool for point-of-care diagnosis (POC) of female breast cancer [108], early identification of prostate cancer relapse [109], and in forensic applications [110]. A biosensor for detection of free prostate-specific antigen (fPSA) using long-range surface plasmon-enhanced fluorescence spectroscopy (LRSP-FS) and photo-cross-linked carboxymethylated dextran hydrogel matrix (shown in Fig. 8a) was reported [111]. As shown by fluorescence kinetics in Fig. 10a, the analyzed sample was firstly flowed over the sensor surface that was functionalized by capture antibodies followed by the binding of fluorophore-labeled detection antibodies. The in situ measured increase of fluorescence signal was proportional to the amount of captured analyte. The sensor allowed the detection of f-PSA in buffer and human serum with the limit of detection (LOD) of $34 \mathrm{fM}$ and $0.33 \mathrm{pM}$, respectively, in $35 \mathrm{~min}$. This LOD was about four orders of magnitude better than that for SPR-based detection 
a

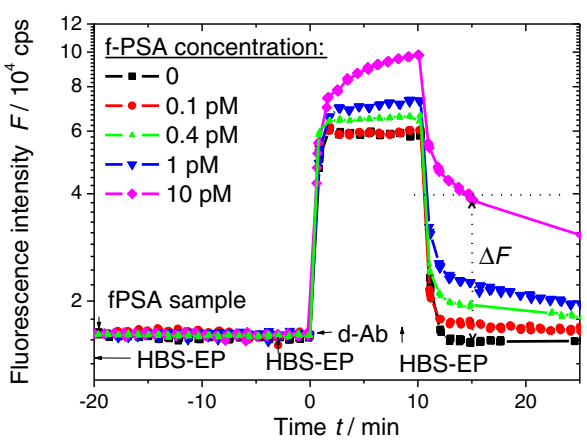

b

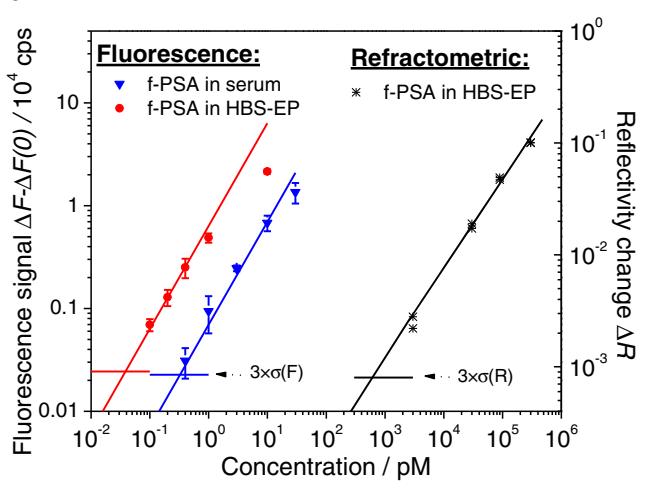

Fig. 10 a Kinetics of fluorescence signal for f-PSA sandwich immunoassay and LRSPP-enhanced fluorescence readout. b Calibration curves for the direct label-free detection of f-PSA (refractometric - stars) are compared to the PEF detection for f-PSA in buffer (circles) and in human serum (triangles) (reproduced with permission from [92])

as can be seen from calibration curves presented in Fig. $10 \mathrm{~b}$. Metallic nanoparticle-enhanced fluorescence assays were developed for the analysis of PSA in female serum in order to perform diagnosis of breast cancer [111]. Sandwich assay format was used for the detection of the concentration ratio of f-PSA and PSA conjugated with $\alpha$-1-anti-chymotrypsin (PSA-ACT) in diluted female serum from healthy personnel and patients with breast cancer. The limit of detection of $\mathrm{f}$ PSA in PBS buffer and diluted female serum was $0.4 \mathrm{pg} / \mathrm{ml}$ $(12 \mathrm{fM})$ and $1.8 \mathrm{pg} / \mathrm{ml}(52 \mathrm{fM})$, respectively, and the analysis required $2 \mathrm{~h}$. Another cancer biomarker-C-reactive protein - was detected by SPFS with sandwich immunoassay. The limit of detection of $26 \mathrm{ng} / \mathrm{ml}(248 \mathrm{pM})$ in human serum diluted 1:20 was reported for $30 \mathrm{~min}$ of analysis time [112]. PEF detection of pancreatic cancer biomarker-UL16-binding protein 2-was implemented to microtiter plate arrays by using a sandwich immunoassay [113]. In this work, the detection antibody was attached to a $25-\mathrm{nm}$ gold nanoparticle and labeled with Atto633 dyes in order to increase the fluorescence signal associated with the binding event. The limit of detection of $18 \mathrm{pg} / \mathrm{ml}(0.75 \mathrm{pM})$ in 1:10 diluted human serum and detection time of about $4.3 \mathrm{~h}$ were reported.

Fluorescence immunoassay for the analysis of human necrosis factor alpha protein (TNF- $\alpha$-immune-modulator agent) was performed by phase-modulation fluorometry amplified by substrates with silver islands [114]. Firstly, the analyzed sample was incubated with a detection antibody labeled with the dye DY488. Afterwards, the mixture was brought in contact with capture antibodies attached to the silver islands, and the fluorescence signal that accompanied the affinity binding was measured. The LOD of $3 \mathrm{pM}$ was reported with the detection time of $2 \mathrm{~h}$. A similar approach was adopted for the detection of troponin I (TnI) which is used as biomarker of myocardial damage [115]. In this work, the sensor chip with silver nanoparticles was subsequently modified with protein A, capture IgG antibody against TnI, and blocked with bovine serum albumin (BSA). Then, the buffer or whole blood sample with TnI was incubated with fluorophore-labeled detection antibody and reacted with the sensor surface. TnI detection in buffer was performed with and without 3-min microwave heating which provided the LOD of $5 \mathrm{pg} / \mathrm{ml}(0.22 \mathrm{pM})$ and $0.1 \mathrm{ng} / \mathrm{ml}(4.3 \mathrm{pM})$, respectively. For whole blood samples, the LOD of $50 \mathrm{pg} / \mathrm{ml}(2.2 \mathrm{pM})$ was obtained when microwave heating was applied. In another example of PEF implementation to microtiter plates, human IgG using was detected by using sandwich immunoassays which for $1 \mathrm{~h}$ incubation time provided LOD of $0.086 \mathrm{ng} / \mathrm{ml}(0.57 \mathrm{pM})$ [116].

\section{Detection of Toxins and Pathogens}

Bacterial pathogens were analyzed based on the detection of specific DNA sequences [117, 118]. Genomic and exosporium DNA of Bacillus anthracis spores were rapidly detected within $1 \mathrm{~min}$ at the microgram per milliliter concentrations by using PEF amplification that utilizes gold nanoclusters [117]. Furthermore, the same work reported the detection of DNA from less than 1,000 vegetative cells in $1 \mathrm{~min}$ by using sensor chips combining the PEF amplification with microwave heating-based extraction of DNA. For the immunoassay detection of whole bacteria, PEF amplification based on tightly confined LSP of SPP fields is not possible due to the large (around a micrometer) size of this type of analyte. Therefore, the SPFS detection principle was combined with the excitation of LRSPPs which exhibit a large penetration depth $L_{\mathrm{p}}$ (see Fig. 9a). This fluorescence readout principle was carried out for E. coli $\mathrm{O} 157: \mathrm{H} 7$ sandwich immunoassay and provided a limit of detection as low as 6 colony forming units (cfu)/ml [119]. The assay was highly specific and required $20 \mathrm{~min}$. Moreover, LRSPP-enhanced fluorescence spectroscopy was adopted for the detection of aflatoxin $\mathrm{M}_{1}\left(\mathrm{AFM}_{1}\right)$ [105]. This harmful low molecular weight analyte is a metabolite of mycotoxin aflatoxin $\mathrm{B}_{1}$ produced mainly by Aspergillus flavus and Aspergillus parasiticus pathogens. The gold sensor surface was functionalized with a conjugate of $\mathrm{AFM}_{1}$ and BSA for the inhibition competitive immunoassay. Monoclonal rat antibody against $\mathrm{AFM}_{1}$ was incubated with a sample containing $\mathrm{AFM}_{1}$, and the unreacted antibody was flowed over the sensor surface and detected by the amplified fluorescence 
spectroscopy. The limit of detection of $\mathrm{AFM}_{1}$ present in milk was determined to be $0.6 \mathrm{pg} / \mathrm{ml}(1.8 \mathrm{pM})$, and the analysis time was $53 \mathrm{~min}$. An assay for severe acute respiratory syndrome (SARS) coronavirus (SARS-CoV) nucleocapsid (GST$\mathrm{N})$ protein was developed with localized surface plasmoncoupled fluorescence fiber optic readout [120]. The sandwich immunoassays enabled the analysis of recombinant SARS$\mathrm{CoV} \mathrm{N}$ protein in a buffer at concentrations as low as $0.1 \mathrm{pg} /$ $\mathrm{ml}$. A similar biosensor platform was employed for the detection of swine-origin influenza A (H1N1) viruses (S-OIV) with the detection limit of $13.9 \mathrm{pg} / \mathrm{ml}$ [121].

\section{Conclusions}

PEF pushed forward the sensitivity and shortened analysis time of assays for the detection of important analytes including biomarkers, pathogens, and toxins. These compounds were detected at low femtomolar concentrations, and the analysis often required only several minutes. We witnessed numerous implementations of this amplification scheme to novel biochips that are compatible with existing microscopy and microarray technologies as well as to entirely new biosensor devices. Up to now, PEF biosensors mostly took advantage of metallic nanostructures providing the amplification of fluorescence intensity by $<10^{2}$. However, current advances in plasmonics paved ways towards much stronger amplifications which can reach factors $>10^{3}$. In order to harness such fluorescence enhancement in practical biosensor technologies, these efforts need to be complemented by the development of new methods for precise and cost-effective fabrication of metallic nanostructures and their selective functionalization in plasmonic hotspots. This review article addresses these challenges and discusses possible future ways in this rapidly developing biosensor field that aims at impacting important areas of point-of-care medical diagnostics, food control, and safety.

Acknowledgments This work was partially supported by the Austrian NANO Initiative (FFG and BMVIT) through the NILPlasmonics project within the NILAustria cluster (www.NILAustria.at) and by the Austrian Science Fund (FWF) through the project ACTIPLAS (P 244920-N20).

\section{References}

1. Hoppener C, Novotny L (2012) Exploiting the light-metal interaction for biomolecular sensing and imaging. Q Rev Biophys 45(2): 209-255

2. Lal S, Grady NK, Kundu J, Levin CS, Lassiter JB, Halas NJ (2008) Tailoring plasmonic substrates for surface enhanced spectroscopies. Chem Soc Rev 37(5):898-911

3. Sharma B, Frontiera RR, Henry AI, Ringe E, Van Duyne RP (2012) SERS: materials, applications, and the future. Mater Today 15(1-2): $16-25$
4. Dostalek J, Knoll W (2008) Biosensors based on surface plasmonenhanced fluorescence spectroscopy. Biointerphases 3(3):FD12FD22

5. Lakowicz JR, Ray K, Chowdhury M, Szmacinski H, Fu Y, Zhang J, Nowaczyk K (2008) Plasmon-controlled fluorescence: a new paradigm in fluorescence spectroscopy. Analyst 133(10):13081346

6. Giljohann DA, Mirkin CA (2009) Drivers of biodiagnostic development. Nature 462(7272):461-464

7. Lazcka O, Del Campo FJ, Munoz FX (2007) Pathogen detection: a perspective of traditional methods and biosensors. Biosens Bioelectron 22(7):1205-1217

8. Ford GW, Weber WH (1984) Electromagnetic interactions of molecules with metal surfaces. Phys Rep 113(4):195-287

9. Giannini V, Fernandez-Dominguez AI, Sonnefraud Y, Roschuk T, Fernandez-Garcia R, Maier SA (2011) Controlling light localization and light-matter interactions with nanoplasmonics. Small 6(22): 2498-2507

10. Giannini V, Fernandez-Dominguez AI, Heck SC, Maier SA (2011) Plasmonic nanoantennas: fundamentals and their use in controlling the radiative properties of nanoemitters. Chem Rev 111(6):38883912

11. Neumann T, Johansson ML, Kambhampati D, Knoll W (2002) Surface-plasmon fluorescence spectroscopy. Adv Funct Mater 12(9):575-586

12. Garcia-Parajo MF (2008) Optical antennas focus in on biology. Nat Photonics 2(4):201-203

13. Fort E, Gresillon S (2008) Surface enhanced fluorescence. J Phys DAppl Phys 41(1)

14. Bharadwaj P, Novotny L (2007) Spectral dependence of single molecule fluorescence enhancement. Opt Express 15(21):14266 14274

15. Drexhage KH (1970) Influence of a dielectric interface on fluorescence decay time. J Lumin 1-2:693-701

16. Benner RE, Dornhaus R, Chang RK (1979) Angular emission profiles of dye molecules excited by surface-plasmon waves at a metal-surface. Opt Commun 30(2):145-149

17. Balanis CA (2005) Antenna theory: analyses and design. Wiley, Hoboken

18. Taminiau TH, Stefani FD, van Hulst NF (2008) Enhanced directional excitation and emission of single emitters by a nano-optical Yagi-Uda antenna. Opt Express 16(14):10858-10866

19. Dregely D, Taubert R, Dorfmuller J, Vogelgesang R, Kern K, Giessen H (2011) 3D optical Yagi-Uda nanoantenna array. Nat Commun 2:1-7

20. West PR, Ishii S, Naik GV, Emani NK, Shalaev VM, Boltasseva A (2010) Searching for better plasmonic materials. Laser Photon Rev 4(6):795-808

21. Ray K, Chowdhury MH, Lakowicz JR (2007) Aluminum nanostructured films as substrates for enhanced fluorescence in the ultraviolet-blue spectral region. Anal Chem 79(17):6480-6487

22. Cortie MB, McDonagh AM (2011) Synthesis and optical properties of hybrid and alloy plasmonic nanoparticles. Chem Rev 111(6): 3713-3735

23. Boltasseva A (2009) Plasmonic components fabrication via nanoimprint. J Opt A-Pure Appl Opt 11(11):11

24. Novotny L, van Hulst N (2011) Antennas for light. Nat Photonics $5(2): 83-90$

25. Halas NJ, Lal S, Chang WS, Link S, Nordlander P (2011) Plasmons in strongly coupled metallic nanostructures. Chem Rev 111(6): 3913-3961

26. Jones MR, Osberg KD, Macfarlane RJ, Langille MR, Mirkin CA (2011) Templated techniques for the synthesis and assembly of plasmonic nanostructures. Chem Rev 111(6):3736-3827

27. Sarid D (1981) Long-range surface-plasma waves on very thin metal-films. Phys Rev Lett 47(26):1927-1930 
28. Barnes WL, Preist TW, Kitson SC, Sambles JR, Cotter NK, Nash DJ (1995) Photonic gaps in the dispersion of surface plasmons on gratings. Phys Rev B 51:11164

29. Toma M, Knoll W, Dostalek J (2011) SPR imaging with high spatial resolution based on Bragg-scattered surface plasmons. Plasmonics 7(2):293-299

30. Masson JF, Murray-Methot MP, Live LS (2010) Nanohole arrays in chemical analysis: manufacturing methods and applications. Analyst 135(7):1483-1489

31. Gordon R, Brolo AG, Sinton D, Kavanagh KL (2010) Resonant optical transmission through hole-arrays in metal films: physics and applications. Laser Photon Rev 4(2):311-335

32. Genevet P, Tetienne JP, Gatzogiannis E, Blanchard R, Kats MA, Scully MO, Capasso F (2010) Large enhancement of nonlinear optical phenomena by plasmonic nanocavity gratings. Nano Lett 10(12):4880-4883

33. Wang DX, Yang T, Crozier KB (2011) Optical antennas integrated with concentric ring gratings: electric field enhancement and directional radiation. Opt Express 19(3):2148-2157

34. Aouani H, Mahboub O, Bonod N, Devaux E, Popov E, Rigneault H, Ebbesen TW, Wenger J (2011) Bright unidirectional fluorescence emission of molecules in a nanoaperture with plasmonic corrugations. Nano Lett 11(2):637-644

35. Sarid D (2010) Modern introduction to surface plasmons: theory, mathematical modeling, and applications. Cambridge University Press, New York

36. Jain PK, Lee KS, El-Sayed IH, El-Sayed MA (2006) Calculated absorption and scattering properties of gold nanoparticles of different size, shape, and composition: applications in biological imaging and biomedicine. J Phys Chem B 110(14):7238-7248

37. Wu DJ, Cheng Y, Liu XJ (2009) "Hot spots" induced near-field enhancements in Au nanoshell and Au nanoshell dimer. Appl Phys B-Lasers Opt 97(2):497-503

38. Shao L, Woo KC, Chen HJ, Jin Z, Wang JF, Lin HQ (2010) Angleand energy-resolved plasmon coupling in gold nanorod dimers. ACS Nano 4(6):3053-3062

39. Harrison RK, Ben-Yakar A (2010) Role of near-field enhancement in plasmonic laser nanoablation using gold nanorods on a silicon substrate. Opt Express 18(21):22556-22571

40. Sasanpour P, Rashidian B, Vossoughi M (2010) Fluorescent microscopy using localized excitation source with gold nanotriangles: a computational study. Photonics Nanostruct 9(3):219-224

41. Talley CE, Jackson JB, Oubre C, Grady NK, Hollars CW, Lane SM, Huser TR, Nordlander P, Halas NJ (2005) Surface-enhanced Raman scattering from individual Au nanoparticles and nanoparticle dimer substrates. Nano Lett 5(8):1569-1574

42. Fischer H, Martin OJF (2008) Engineering the optical response of plasmonic nanoantennas. Opt Express 16(12):9144-9154

43. Kinkhabwala A, Yu ZF, Fan SH, Avlasevich Y, Mullen K, Moerner WE (2009) Large single-molecule fluorescence enhancements produced by a bowtie nanoantenna. Nat Photonics 3(11):654 657

44. Kern AM, Martin OJF (2011) Excitation and reemission of molecules near realistic plasmonic nanostructures. Nano Lett 11(2):482487

45. Lamprecht B, Schider G, Lechner RT, Ditlbacher H, Krenn JR, Leitner A, Aussenegg FR (2000) Metal nanoparticle gratings: influence of dipolar particle interaction on the plasmon resonance. Phys Rev Lett 84(20):4721-4724

46. Parsons J, Hendry E, Burrows CP, Auguie B, Sambles JR, Barnes WL (2009) Localized surface-plasmon resonances in periodic nondiffracting metallic nanoparticle and nanohole arrays. Phys Rev B 79(7)

47. Johnson WL, Kim SA, Utegulov ZA, Shaw JM, Draine BT (2009) Optimization of arrays of gold nanodisks for plasmon-mediated Brillouin light scattering. J Phys Chem C 114:14651-14657
48. Xie F, Pang JS, Centeno A, Ryan MP, Riley DJ, Alford NM (2013) Nanoscale control of Ag nanostructures for plasmonic fluorescence enhancement of near-infrared dyes. Nano Res 6(7):496-510

49. Auguie B, Bendana XM, Barnes WL, de Abajo FJG (2010) Diffractive arrays of gold nanoparticles near an interface: critical role of the substrate. Phys Rev B 82(15):7

50. Chu YZ, Schonbrun E, Yang T, Crozier KB (2008) Experimental observation of narrow surface plasmon resonances in gold nanoparticle arrays. Appl Phys Lett 93(18):3

51. Vecchi G, Giannini V, Rivas JG (2009) Surface modes in plasmonic crystals induced by diffractive coupling of nanoantennas. Phys Rev B 80(20):4

52. Zhou W, Odom TW (2011) Tunable subradiant lattice plasmons by out-of-plane dipolar interactions. Nat Nanotechnol 6(7): 423-427

53. Bauch M, Dostalek J (2013) Collective localized surface plasmons for high performance fluorescence biosensing. Opt Express 21(17): 20470-20483

54. Weber WH, Eagen CF (1979) Energy-transfer from an excited dye molecule to the surface-plasmons of an adjacent metal. Opt Lett 4(8):236-238

55. Knoll W, Philpott MR, Swalen JD (1981) Emission of light from Ag metal gratings coated with dye monolayer assemblies. J Chem Phys 75(10):4795-4799

56. Stefani FD, Vasilev K, Bocchio N, Stoyanova N, Kreiter M (2005) Surface-plasmon-mediated single-molecule fluorescence through a thin metallic film. Phys Rev Lett 94(2)

57. Punj D, Mivelle M, Moparthi SB, van Zanten TS, Rigneault H, van Hulst NF, Garcia-Parajo MF, Wenger J (2013) A plasmonic 'antenna-in-box' platform for enhanced single-molecule analysis at micromolar concentrations. Nat Nanotechnol 8(7):512-516

58. Attridge JW, Daniels PB, Deacon JK, Robinson GA, Davidson GP (1991) Sensitivity enhancement of optical immunosensors by the use of a surface-plasmon resonance fluoroimmunoassay. Biosens Bioelectron 6(3):201-214

59. Kasry A, Knoll W (2006) Long range surface plasmon fluorescence spectroscopy. Appl Phys Lett 89(10):101106

60. Dostalek J, Kasry A, Knoll W (2007) Long range surface plasmons for observation of biomolecular binding events at metallic surfaces. Plasmonics 2(3):97-106

61. Huang CJ, Dostalek J, Knoll W (2010) Optimization of layer structure supporting long range surface plasmons for surface plasmon-enhanced fluorescence spectroscopy biosensors. J Vac Sci Technol B 28(1):66-72

62. Lakowicz JR, Malicka J, Gryczynski I, Gryczynski Z (2003) Directional surface plasmon-coupled emission: a new method for high sensitivity detection. Biochem Biophys Res Commun 307(3): 435-439

63. Yuk JS, Trnavsky M, McDonagh C, MacCraith BD (2010) Surface plasmon-coupled emission (SPCE)-based immunoassay using a novel paraboloid array biochip. Biosens Bioelectron 25(6):1344 1349

64. Toma K, Vala M, Adam P, Homola J, Knoll W, Dostalek J (2013) Compact biochip for plasmon-enhanced fluorescence biochip. Opt Express 21(8): 10121-10132

65. Toma K, Dostalek J, Knoll W (2011) Long range surface plasmoncoupled fluorescence emission for biosensor applications. Opt Express 19(12):11090-11099

66. Toma M, Toma K Adam P, Vala M, Homola J, Knoll W, Dostalek J (2012) Surface plasmon-coupled emission on plasmonic Bragg gratings. Opt Express 20(13):14042-14053

67. Kitson SC, Barnes WL, Sambles JR, Cotter NPK (1996) Excitation of molecular fluorescence via surface plasmon polaritons. J Mod Optic 43(3):573-582

68. Tawa K, Cui XQ, Kintaka K, Nishii J, Morigaki K (2011) Sensitive bioimaging in microfluidic channels on the plasmonic substrate: 
application of an enhanced fluorescence based on the reverse coupling mode. J Photochem Photobiol A-Chem 221(2-3):261-267

69. Aouani H, Mahboub O, Devaux E, Rigneault H, Ebbesen TW, Wenger J (2011) Plasmonic antennas for directional sorting of fluorescence emission. Nano Lett 11(6):2400-2406

70. Aroca R, Kovacs G, Jennings C, Loutfy R, Vincett P (1988) Fluorescence enhancement from Langmuir-Blodgett monolayers on silver island films. Langmuir 4(3):518-521

71. Matveeva EG, Gryczynski Z, Lakowicz JR (2005) Myoglobin immunoassay based on metal particle-enhanced fluorescence. J Immunol Methods 302(1-2):26-35

72. Touahir L, Galopin E, Boukherroub R, Gouget-Laemmel AC, Chazalviel JN, Ozanam F, Szunerits S (2010) Localized surface plasmon-enhanced fluorescence spectroscopy for highly-sensitive real-time detection of DNA hybridization. Biosens Bioelectron 25(12):2579-2585

73. Fan JA, Wu CH, Bao K, Bao JM, Bardhan R, Halas NJ, Manoharan VN, Nordlander P, Shvets G, Capasso F (2010) Self-assembled plasmonic nanoparticle clusters. Science 328(5982):1135-1138

74. Liu SY, Huang L, Li JF, Wang C, Li Q, Xu HX, Guo HL, Meng ZM, Shi Z, Li ZY (2013) Simultaneous excitation and emission enhancement of fluorescence assisted by double plasmon modes of gold nanorods. J Phys Chem C 117(20):10636-10642

75. Gill R, Tian LJ, Somerville WRC, Le Ru EC, van Amerongen H, Subramaniam V (2012) Silver nanoparticle aggregates as highly efficient plasmonic antennas for fluorescence enhancement. J Phys Chem C 116(31):16687-16693

76. Schmelzeisen M, Zhao Y, Klapper M, Muellen K, Kreiter M (2010) Fluorescence enhancement from individual plasmonic gap resonances. ACS Nano 4(6):3309-3317

77. Curto AG, Volpe G, Taminiau TH, Kreuzer MP, Quidant R, van Hulst NF (2010) Unidirectional emission of a quantum dot coupled to a nanoantenna. Science 329(5994):930-933

78. Zhang W, Ding F, Li W, Wang Y, Hu J, Chou SY (2012) Giant and uniform fluorescence enhancement over large areas using plasmonic nanodots in $3 \mathrm{D}$ resonant cavity nanoantenna by nanoimprinting. Nanotechnology 23:225301

79. Yoo HW, Jung JM, Lee SK, Jung HT (2011) The fabrication of highly ordered silver nanodot patterns by platinum assisted nanoimprint lithography. Nanotechnology 22(9):7

80. Zin MT, Leong K, Wong N-Y, Ma H, Sarikaya M, Jen AKY (2009) Surface-plasmon-enhanced fluorescence from periodic quantum dot arrays through distance control using biomolecular linkers. Nanotechnology 20(1)

81. Geyer S, Porter VJ, Halpert JE, Mentzel TS, Kastner MA, Bawendi MG (2010) Charge transport in mixed CdSe and CdTe colloidal nanocrystal films. Phys Rev B 82(15):8

82. Brolo AG, Kwok SC, Moffitt MG, Gordon R, Riordon J, Kavanagh KL (2005) Enhanced fluorescence from arrays of nanoholes in a gold film. J Am Chem Soc 127(42):14936-14941

83. Samanta D, Sarkar A (2011) Immobilization of bio-macromolecules on self-assembled monolayers: methods and sensor applications. Chem Soc Rev 40(5):2567-2592

84. Mateescu A, Wang Y, Dostalek J, Jonas U (2012) Thin hydrogel films for optical biosensor applications. Membranes 2(1):40-69

85. Love JC, Estroff LA, Kriebel JK, Nuzzo RG, Whitesides GM (2005) Self-assembled monolayers of thiolates on metals as a form of nanotechnology. Chem Rev 105(4):1103-1169

86. Gooding JJ, Ciampi S (2011) The molecular level modification of surfaces: from self-assembled monolayers to complex molecular assemblies. Chem Soc Rev 40(5):2704-2718

87. Vericat C, Vela ME, Benitez G, Carro P, Salvarezza RC (2010) Selfassembled monolayers of thiols and dithiols on gold: new challenges for a well-known system. Chem Soc Rev 39(5):1805-1834

88. Tawa K, Yokota Y, Kintaka K, Nishii J, Nakaoki T (2011) An application of a plasmonic chip with enhanced fluorescence to a simple biosensor with extended dynamic range. Sensors Actuators B-Chem 157(2):703-709

89. Huber C, Liu J, Egelseer EM, Moll D, Knoll W, Sleytr UB, Sara M (2006) Heterotetramers formed by an S-layer-streptavidin fusion protein and core-streptavidin as a nanoarrayed template for biochip development. Small 2(1):142-150

90. Vollenkle C, Weigert S, Ilk N, Egelseer E, Weber V, Loth F, Falkenhagen D, Sleytr UB, Sara M (2004) Construction of a functional S-layer fusion protein comprising an immunoglobulin Gbinding domain for development of specific adsorbents for extracorporeal blood purification. Appl Environ Microbiol 70(3):1514 1521

91. Nomellini JF, Duncan G, Dorocicz IR, Smit J (2007) S-layermediated display of the immunoglobulin G-binding domain of streptococcal protein $\mathrm{G}$ on the surface of Caulobacter crescentus: development of an immunoactive reagent. Appl Environ Microbiol 73(10):3245-3253

92. Wang Y, Brunsen A, Jonas U, Dostalek J, Knoll W (2009) Prostate specific antigen biosensor based on long range surface plasmonenhanced fluorescence spectroscopy and dextran hydrogel binding matrix. Anal Chem 81(23):9625-9632

93. Yu F, Persson B, Lofas S, Knoll W (2004) Attomolar sensitivity in bioassays based on surface plasmon fluorescence spectroscopy. J Am Chem Soc 126(29):8902-8903

94. Ray K, Badugu R, Lakowicz JR (2007) Polyelectrolyte layer-bylayer assembly to control the distance between fluorophores and plasmonic nanostructures. Chem Mater 19(24):5902-5909

95. Piliarik M, Kvasnicka P, Galler N, Krenn JR, Homola J (2011) Local refractive index sensitivity of plasmonic nanoparticles. Opt Express 19(10):9213-9220

96. Feuz L, Jonsson P, Jonsson MP, Hook F (2010) Improving the limit of detection of nanoscale sensors by directed binding to highsensitivity areas. ACS Nano 4(4):2167-2177

97. Alvarez M, Best A, Unger A, Alonso JM, del Campo A, Schmelzeisen M, Koynov K, Kreiter M (2010) Near-field lithography by two-photon-induced photocleavage of organic monolayers. Adv Funct Mater 20(24):4265-4272

98. Caswell KK, Wilson JN, Bunz UHF, Murphy CJ (2003) Preferential end-to-end assembly of gold nanorods by biotin-streptavidin connectors. J Am Chem Soc 125(46):13914-13915

99. Fu Y, Zhang J, Lakowicz JR (2010) Plasmon-enhanced fluorescence from single fluorophores end-linked to gold nanorods. J Am Chem Soc 132(16):5540-5541

100. Rathanaswami P, Roalstad S, Roskos L, Su QJ, Lackie S, Babcook J (2005) Demonstration of an in vivo generated sub-picomolar affinity fully human monoclonal antibody to interleukin- 8 . Biochem Biophys Res Commun 334(4):1004-1013

101. Rispens T, Ooijevaar-de Heer PD, Derksen NI, Wolbink G, van Schouwenburg P, Kruithof S, Aalberse R (2013) Nanomolar to subpicomolar affinity measurements of antibody-antigen interactions and protein multimerizations: fluorescence-assisted high-performance liquid chromatography. Anal Biochem 437(2):118-122

102. Aslan K, Zhang Y, Geddes CD (2009) Sonication-assisted metalenhanced fluorescence (SAMEF)-based bioassays. Anal Chem 81: 4713-4719

103. Aslan K, Malyn SN, Geddes CD (2006) Microwave-accelerated surface plasmon-coupled directional luminescence: application to fast and sensitive assays in buffer, human serum and whole blood. $\mathrm{J}$ Immunol Methods 323:55-64

104. Vasdekis AE, Laporte GPJ (2011) Enhancing single molecule imaging in optofluidics and microfluidics. Int J Mol Sci 12:5135-5156

105. Wang Y, Dostalek J, Knoll W (2009) Biosensor for detection of aflatoxin $\mathrm{M}_{1}$ in milk based on long range surface plasmon enhanced fluorescence spectroscopy. Biosens Bioelectron 24(7):2264-2267

106. Yuk JS, MacCraith BD, McDonagh C (2011) Signal enhancement of surface plasmon-coupled emission (SPCE) with the evanescent 
field of surface plasmons on a bimetallic paraboloid biochip. Biosens Bioelectron 26(7):3213-3218

107. Zhou L, Ding F, Chen H, Ding W, Zhang W, Chou S-Y (2012) Enhancement of immunoassay's fluorescence and detection sensitivity using three-dimensional plasmonic nano-antenna-dots array. Anal Chem 84:4489-4495

108. Radowicki S, Kunicki M, Bandurska-Stankiewicz E (2008) Prostate-specific antigen in the serum of women with benign breast disease. Eur J Obstet Gynecol Reprod Biol 138(2):212-216

109. Doherty AP, Bower M, Smith GL, Miano R, Mannion EM, Mitchell H, Christmas TJ (2000) Undetectable ultrasensitive PSA after radical prostatectomy for prostate cancer predicts relapse-free survival. Br J Cancer 83(11):1432-1436

110. Johnson ED, Kotowski TM (1993) Detection of prostate specific antigen by ELISA. J Forensic Sci 38(2):250-258

111. Chang Y-F, Hung S-H, Lee Y-J, Chen R-C, Su L-C, Lai C-S, Chou C (2011) Discrimination of breast cancer by measuring prostatespecific antigen levels in women's serum. Anal Chem 83(13):5324 5328

112. Scholten A, Menges B, Juebner M, Rothschild MA, Bender K (2013) A mixed alkanethiol based immunosensor for surface plasmon field-enhanced fluorescence spectroscopy in serum. Analyst 138:1705

113. Chang Y-F, Yu J-S, Chang Y-T, Su L-C, Wu C-C, Chang Y-S, Lai SS, Chou C (2013) The utility of a high-throughput scanning biosensor in the detection of the pancreatic cancer marker ULBP2. Biosens Bioelectron 43:232-237

114. Szmacinski H, Murtaza Z, Lakowicz JR (2010) Time-resolved fluorometric method for one-step immunoassays using plasmonic nanostructures. J Phys Chem C Nanomater Interfaces 114(16): 7236-7241

115. Aslan K, Grell TAJ (2011) Rapid and sensitive detection of troponin I in human whole blood samples by using silver nanoparticle films and microwave heating. Clin Chem 57(5):746-752

116. Nooney R, Clifford A, LeGuevel X, Stranik O, McDonagh C, MacCraith BD (2010) Enhancing the analytical performance of immunoassays that employ metal-enhanced fluorescence. Anal Bioanal Chem 396(3):1127-1134

117. Aslan K, Zhang Y, Hibbs S, Baillie L, Previt MJR, Geddes CD (2007) Microwave-accelerated metal-enhanced fluorescence: application to detection of genomic and exosporium anthrax DNA in< 30 seconds. Analyst 132:1130-1138

118. Aslan K, Previte MJR, Zhang YX, Gallagher T, Baillie L, Geddes CD (2008) Extraction and detection of DNA from Bacillus anthracis spores and the vegetative cells within $1 \mathrm{~min}$. Anal Chem 80(11):4125-4132

119. Huang CJ, Sessitsch A, Dostalek J, Knoll W (2011) Long range surface plasmon-enhanced fluorescence spectroscopy biosensor for ultrasensitive detection of E. coli O157:H7. Anal Chem 83(3):674-677

120. Huang JC, Chang Y-F, Chen K-H, Su L-C, Lee C-W, Chen C-C, Chen Y-MA, Chou C (2009) Detection of severe acute respiratory syndrome (SARS) coronavirus nucleocapsid protein in human serum using a localized surface plasmon coupled fluorescence fiber-optic biosensor. Biosens Bioelectron 25(2):320-325

121. Chang Y-F, Wang S-F, Huang JC, Su L-C, Yao L, Li Y-C, Wu S-C, Chen Y-MA, Hsieh J-P, Chou C (2010) Detection of swine-origin influenza A (H1N1) viruses using a localized surface plasmon coupled fluorescence fiber-optic biosensor. Biosens Bioelectron 26(3):1068-1073

122. Hwang E, Smolyaninov II, Davis CC (2010) Surface plasmon polariton enhanced fluorescence from quantum dots on nanostructured metal surfaces. Nano Lett 10(3):813-820

123. Tawa K, Hori H, Kintaka K, Kiyosue K, Tatsu Y, Nishii J (2008) Optical microscopic observation of fluorescence enhanced by grating-coupled surface plasmon resonance. Opt Express 16(13): 9781-9790

124. Jiang Y, Wang HY, Wang H, Gao BR, Hao YW, Jin Y, Chen QD, Sun HB (2011) Surface plasmon enhanced fluorescence of dye molecules on metal grating films. J Phys Chem C 115(25):1263612642

125. Renier A, Mangeat T, Benalia H, Elie-Caille C, Pieralli C, Wacogne B (2010) Gold/silica thin film for biosensors applications: metal enhanced fluorescence. Laser Phys 20(3):591-595

126. Xu ZD, Chen Y, Gartia MR, Jiang J, Liu GL (2011) Surface plasmon enhanced broadband spectrophotometry on black silver substrates. Appl Phys Lett 98(24):3

127. Volpati D, Job AE, Aroca RF, Constantino CJL (2008) Molecular and morphological characterization of bis benzimidazo perylene films and surface-enhanced phenomena. J Phys Chem B 112(13): 3894-3902

128. Bharadwaj P, Novotny L (2010) Plasmon-enhanced photoemission from a single Y(3)N@C(80) fullerene. J Phys Chem C 114(16): 7444-7447

129. Fu Y, Zhang J, Lakowicz JR (2012) Large enhancement of single molecule fluorescence by coupling to hollow silver nanoshells. Chem Commun 48(78):9726-9728

130. Guerrero AR, Zhang Y, Aroca RF (2012) Experimental confirmation of local field enhancement determining far-field measurements with shell-isolated silver nanoparticles. Small 8(19):2964-2967

131. Zhang WH, Ding F, Li WD, Wang YX, Hu J, Chou SY (2012) Giant and uniform fluorescence enhancement over large areas using plasmonic nanodots in $3 \mathrm{D}$ resonant cavity nanoantenna by nanoimprinting. Nanotechnology 23(22):9

132. Yu YM, Feng CL, Caminade AM, Majoral JP, Knoll W (2009) The detection of DNA hybridization on phosphorus dendrimer multilayer films by surface plasmon field enhanced-fluorescence spectroscopy. Langmuir 25(23):13680-13684

133. Aslan K, Huang J, Wilson GM, Geddes CD (2006) Metal-enhanced fluorescence-based RNA sensing. J Am Chem Soc 128(13):42064207

134. Dragan AI, Albrecht MT, Pavlovic R, Keane-Myers AM, Geddes CD (2012) Ultra-fast pg/ml anthrax toxin (protective antigen) detection assay based on microwave-accelerated metal-enhanced fluorescence. Anal Biochem 425:54-61 